Review Article

\title{
Phytochemistry and Biological Activities of Poria
}

\author{
Jie Lu $\mathbb{D},{ }^{1}$ Jing Tian $\left(\mathbb{D},{ }^{1}\right.$ Li Zhou $\left(\mathbb{D},{ }^{1}\right.$ Lijun Meng $\left(\mathbb{D},{ }^{1}\right.$ Sitan Chen $\left(\mathbb{D},{ }^{1}\right.$ Changyang Ma $\mathbb{D},{ }^{1,2,3}$ \\ Jinmei Wang $\mathbb{D},{ }^{1,2,3}$ Zhenhua Liu $\mathbb{D}^{1,2,3}$ Changqin Li $\mathbb{D}^{1,2,3}$ and Wenyi Kang $\mathbb{D}^{1,2,3}$
}

\author{
${ }^{1}$ National $R$ \& D Center for Edible Fungus Processing Technology, Henan University, Kaifeng 475004, Henan, China \\ ${ }^{2}$ Joint International Research Laboratory of Food \& Medicine Resource Function, Kaifeng 475004, Henan, China \\ ${ }^{3}$ Functional Food Engineering Technology Research Center, Kaifeng 475004, Henan, China
}

Correspondence should be addressed to Zhenhua Liu; liuzhenhua623@163.com, Changqin Li; lcq@vip.henu.edu.cn, and Wenyi Kang; kangweny@hotmail.com

Received 20 December 2020; Revised 11 March 2021; Accepted 17 March 2021; Published 29 March 2021

Academic Editor: Wagdy Eldehna

Copyright (C) 2021 Jie Lu et al. This is an open access article distributed under the Creative Commons Attribution License, which permits unrestricted use, distribution, and reproduction in any medium, provided the original work is properly cited.

\begin{abstract}
Poria is a common Traditional Chinese Medicine in clinic. In recent years, the chemical and pharmacological studies of Poria have made great progress, triterpenes and polysaccharides have been isolated, and various types of compounds containing lipids, octanoic acids, fatty acids, and trace elements have been found. In this paper, we reviewed the literature, summarized the main compound types, and reviewed in detail their pharmacological effects in antitumor, immunomodulatory, effects on kidney, hepatoprotective activity, effects on blood sugar, antioxidant effects, anti-inflammatory effects, effects on the gut, antidepressant, and so on, and also categorized the compounds with the same or similar pharmacological effects to provide a reference for the indepth study of the material basis of the pharmacological effect, quality standards, and pharmacological activity of Poria.
\end{abstract}

\section{Introduction}

Poria is the dry sclerotia of Basidiomycota, Agaricomycetes, Polyporaceae, and fungus Poria cocos (Schw.) Wolf. It is cultivated in many regions of China, mainly in Anhui, Yunnan, and Hubei Province [1, 2]. Poria has a long history, which was the first recorded in Shennong Materia Medica $[3,4]$. During the Qin and Han Dynasties, people often used Poria as a tea drink with the belief that it could prolong life $[5,6]$.

So far, the triterpenes, polysaccharides, sterols, fatty acids, and volatile oil are the main chemical components found in Poria, and the triterpenes and polysaccharides are the most active compounds, according to previous pharmacological studies. Moreover, pharmacological research showed that Poria had antitumor, antioxidation, anti-inflammation, diuresis, immune regulation, and intestinal regulation $[2,7]$. It is also used to treat uterine fibroids, chronic pelvic inflammatory disease, endometriosis, ovarian cyst, early pregnancy abortion, menstrual abdominal pain, medical abortion, cold, headache, colitis, arthritis, urinary tract infection, nephritis, and some tumor diseases, such as esophageal cancer and ovarian cancer $[7,8]$. Therefore, this paper summarized the chemical composition and pharmacological activity of Poria based on comprehensive literature analysis, hoping to provide a reference for the indepth research of scientific researchers and the clinical application of medical workers.

\section{Chemical Composition Study}

The chemical composition of Poria was collected from relevant literature through Web of Science, Wanfang.com, CNKI.com, BaiduAcademic.com, and Duxiu.com. Compounds obtained from Poria during the period from 1939 to 2020 were collected and classified according to the structural type. It is evidenced that Poria contains terpenes, sterols, pachymaran, fatty acids, volatile oil, and some inorganic elements, and the effective active components are mainly triterpenes and pachymaran. The triterpenes make up most of the Poria [9], while pachymaran accounts for the majority of the sclerotia (over 80\%) in dry Poria [7]. 
2.1. Terpenoids. In 1939, Japanese scientists boiled the mixture of Poria with pyridine, acetic anhydride, and some other compounds in methanol and obtained one triterpene substance, pachymic acid (1) [10]. Subsequently, a new triterpene compound, dehydrotrametenolic acid (39), was found in Poria sclerotia in 1970 [11], and ganoderic acid (11) was isolated from Poria powder in 1998 [12]. So far, a total of 163 terpenoids have been identified, and they are mainly triterpenoids, diterpenoids, and sterols. According to the basic skeleton, 4 categories of terpenoids in Poria, lanoster8-ene triterpenes, lanoster-7,9(11)-diene triterpenes, 3,4ring-opening lanoster-8-ene triterpenes, and 3,4-ringopening lanoster-7,9(11)-diene triterpenes [13] are separated as shown in Table 1. Besides, their molecular structures are presented in Figure 1.

2.2. Polysaccharides. Polysaccharides account for $70 \% \sim 90 \%$ of the dry weight of Poria, and until now, 35 kinds of PAC from Poria have been reported (Table 2) [63]. Many scholars have found that the physicochemical properties and biological activities of polysaccharides would change to a certain extent after the chemical modification or the introduction of some specific chemical groups [64], which are taken as one efficient way to treat some human diseases [65-67].

2.3. Other Compounds. Besides triterpenes and polysaccharides, there are some other components in Poria, mainly including octanoic acid, lauric acid, undecanoic acid, fatty acid, palmitic acid, carotene, choline, adenine $[1,16]$, and some inorganic elements such as calcium, magnesium, iron, sodium, manganese, etc.

\section{Bioactivity of Poria}

In this paper, articles related to the pharmacological activity of Poria after 2011 were selected as references. And its pharmacological effects are summarized (Table 3).

3.1. Antitumor Action. The antitumor effect of Poria has been attracting many researchers for a long time. Many studies found that triterpenes and polysaccharides in Poria had obvious antitumor activity, especially to the colon cancer cells, lung adenocarcinoma cells, kidney cancer cells, human prostate cancer cells, cervical cancer cells, and human breast cancer cells.

3.1.1. The Antitumor Effect of Poria Triterpenes. Lin proved that total triterpenes of Poria had a significant inhibitory effect on the RKO cell line by inducing apoptosis through caspase 9 and caspase 3 activated by the combination of Cyt $\mathrm{C}$ and Apaf-1 [68]. Triterpenes from Poria could inhibit the proliferation of the A549 cell line by increasing the protein expression levels of Nrf2, GST, and NQO1 [69]. PA induced apoptosis of human renal cell carcinoma 786-0 cell line by inhibiting the activation of the Wnt signaling pathway [70]. PA could inhibit the expression of trim $29 \mathrm{mRNA}$, activate caspase-9, and inhibit the expression of cyclin D1, which indicated that PA could induce apoptosis of Caski cells through inhibition [71]. Jiang found that PA could promote the apoptosis of human breast cancer MDA-MB-231 cells by enhancing the activity of caspase and the expression of cleaved PARP [72]. Poria ethanol extract could induce apoptosis by decreasing the expression of $\mathrm{Bcl}-2$ and increasing the expression of Bax, increasing the content of cytoplasm, the active forms of cleaved caspase- 9 and caspase-3, and cleaved PARP, which proved that pachymic acid had an inhibitory effect on MDA-MB-231 cells. Poria ethanol extract treatment alleviated the damage to the liver and normalized the serum levels of ALT and AST in mice compared with the mice with cisplatin treatment [73]. The study showed that PA could induce apoptosis of SGC-7901 cells by inactivating the JAK2/STAT3 signaling pathway, which proved that PA was a potential bioactive substance for the treatment of gastric cancer [74]. PA could induce caspase 3-mediated apoptosis of HOS and primary osteosarcoma cells by increasing PTEN expression and inhibiting Akt activation [75].

3.1.2. The Antitumor Effect of PAC. Tang proved that PAC could inhibit the phosphorylation of the ERK signaling pathway by downregulating the expression of $p$-ERK1/2, which indicated that PAC could inhibit the proliferation and promote apoptosis of HeLa cells [52]. Lin et al. demonstrated that FMGP inhibited the migration of lung cancer CL1-5 cells by downregulating TGF $\beta$ RI expression and simultaneously decreasing the phosphorylation levels of FAK and Akt [53]. CMP3 induced HepG2 cell apoptosis through two pathways. The first pathway was to promote HepG2 cell apoptosis by upregulating the release of pro-apoptotic proteins Bax, Caspase-3, p53, and cyto C. The other pathway was to upregulate the expression of Fas, FasL, and FADD mRNA, and promote the expression of caspase-3, caspase- 8 , and caspase-9 [54]. PPSW-1 and Sul-W-1 inhibit the migration of MDA-MB-231 cells by inhibiting SATB1 gen [56].

In summary, Poria plays an antitumor role mainly by inhibiting tumor cell proliferation, inducing cell apoptosis, and inhibiting tumor cell metastasis.

3.2. Immune Regulation. Poria also has immunological activity in vitro and in vivo. Triterpenes and polysaccharides from Poria were found with extensive immunomodulatory effects and could improve the immune function of the body. Xie et al. study showed that total triterpenes of Poria could reduce the metabolic activity of spleen cells in mice stimulated by LPS and Con A and reduce the levels of IgG, IgM, IL-2, and IFN- $\gamma$. Total triterpenes can reduce the levels of serum hemolysin and IL-4 in humoral immune response model mice. The spleen index was decreased in high doses (400 mg/ $\mathrm{kg}$ ) and medium doses $(200 \mathrm{mg} / \mathrm{kg})$, indicating that total triterpenes had inhibitory effects on the immune function of mice in vitro and in vivo [76]. Wang et al. study showed that S-CMP could significantly reduce the content of MDA and significantly increase the titer of serum hemolysin antibody and the production of spleen antibody. This 
Table 1: Terpenoids isolated from Poria.

\begin{tabular}{|c|c|c|}
\hline No. & Name & Ref. \\
\hline \multicolumn{3}{|c|}{ Lanoster-8-ene triterpenes $(1-38)$} \\
\hline (1) & Pachymic acid & {$[14]$} \\
\hline (2) & Tumulosic acid & {$[14]$} \\
\hline (3) & Trametenolic acid & {$[9]$} \\
\hline (4) & Eburicoic acid & {$[9]$} \\
\hline (5) & 3-O-acetyl-16 $\alpha$-hydroxytrametenolic acid & {$[15]$} \\
\hline (6) & $16 \alpha$-Hydroxytrametenolic acid & {$[16]$} \\
\hline (7) & O-acetylpachymic acid & {$[12]$} \\
\hline (8) & O-acetylpachymic acid-25-ol & {$[12]$} \\
\hline (9) & Methyl-O-acetylpachymate & {$[12]$} \\
\hline (10) & Pachymic acid methyl ester & {$[12]$} \\
\hline (11) & Ganoderic acid & {$[12]$} \\
\hline (12) & 25-Hydroxypachymic acid & {$[16]$} \\
\hline (13) & 25-Hydroxy-3-epitumulosic acid & {$[17]$} \\
\hline$(14)$ & $16 \alpha, 25$-Dihydroxyeburiconic acid & {$[17]$} \\
\hline$(15)$ & $16 \alpha$-Hydroxyeburiconic acid & {$[17]$} \\
\hline$(16)$ & $3 \beta, 16 \alpha$-Dihydroxy-7-oxo-24-methyllanosta-8,24(31)-dien-21-oic acid & {$[18]$} \\
\hline (17) & $3 \alpha, 16 \alpha$-Dihydroxy-7-oxo-24-methyllanosta-8,24(31)-dien-21-oic acid & {$[18]$} \\
\hline$(18)$ & Oxotrametenolic acid & {$[19]$} \\
\hline (19) & Acetyl eburicoic acid & {$[20]$} \\
\hline$(20)$ & Poricoic acid $\mathrm{ZH}$ & {$[2]$} \\
\hline$(21)$ & Poricoic acid ZU & {$[2]$} \\
\hline$(22)$ & Poricoic acid ZW & {$[2]$} \\
\hline$(23)$ & $3 \beta, 15 \alpha$-Dihydroxy-24-oxolanosta-8-en- 21-oic acid & {$[16]$} \\
\hline$(24)$ & $3 \beta$-Acetyloxy-16 $\alpha$-hydroxy-24-oxolanost-8-en-21-oic acid & {$[16]$} \\
\hline$(25)$ & Daedaleanic acid B & {$[16]$} \\
\hline$(26)$ & $15 \alpha$-Hydroxyeburiconic acid & {$[16]$} \\
\hline$(27)$ & $3 \alpha, 16 \alpha, 25$-Trihydroxylanosta-8,24-dien- 21-oic acid & {$[16]$} \\
\hline$(28)$ & $16 \alpha, 29$-Dihydroxyeburiconic acid & {$[16]$} \\
\hline$(29)$ & $3 \beta$-Acetyloxy-16 $\alpha, 26$-dihydroxylanosta-8,24-dien-21-oic acid & {$[16]$} \\
\hline$(30)$ & $15 \alpha$-Hydroxy-3-oxolanosta-8,24-dien-21-oic acid & {$[16]$} \\
\hline$(31)$ & $16 \alpha$-Hydroxy-3-oxolanosta-8,24-dien-21-oic acid & {$[16]$} \\
\hline$(32)$ & $3 \beta, 16 \alpha$-Bis(acetyloxy)-29-hydroxylanosta-8,24-dien-21-oic acid & {$[16]$} \\
\hline$(33)$ & Hispindic acid B & {$[16]$} \\
\hline$(34)$ & $3 \beta, 15 \alpha$-Bis(acetyloxy)-24- methylenelanost-8-en-21-oic acid & {$[16]$} \\
\hline$(35)$ & $16 \alpha$-Acetyloxyeburiconic acid & {$[16]$} \\
\hline$(36)$ & 3-Epi-pachymic acid & {$[16]$} \\
\hline (37) & Ceanphytamic acid A & {$[21]$} \\
\hline$(38)$ & Ceanphytamic acid B & {$[21]$} \\
\hline \multicolumn{3}{|c|}{ Lanoster-7,9(11)-diene triterpenes (39-82) } \\
\hline$(39)$ & Dehydrotrametenolic acid & {$[14]$} \\
\hline$(40)$ & Dehydropachymic acid & {$[22]$} \\
\hline$(41)$ & Dehydroeburicoic acid & [9] \\
\hline$(42)$ & $6 \alpha$-Hydroxypolyporenic acid C & {$[2]$} \\
\hline (43) & 3-Epi-dehydrotumulosic acid & {$[15]$} \\
\hline$(44)$ & 25-Hydroxy-3-epi-dehydrotumulosic acid & {$[15]$} \\
\hline$(45)$ & Dehydrotumulosic acid & {$[15]$} \\
\hline (46) & Dehydroeburiconic acid & {$[15]$} \\
\hline$(47)$ & 3-O-Acetyl-16 $\alpha$-hydroxydehydrotrametenolic acid & {$[15]$} \\
\hline$(48)$ & 3-Epidehydropachymic acid & {$[15]$} \\
\hline$(49)$ & $3 \beta, 16 \alpha$-Dihydroxylanosta-7,9(11),24-trien-21-oic acid & {$[23]$} \\
\hline$(50)$ & $6 \alpha$-Hydroxydehydropachymic acid & {$[16]$} \\
\hline$(51)$ & $3 \beta$-p-Hydroxybenzoyldehydrotumulosic acid & {$[24]$} \\
\hline$(52)$ & $3 \beta$-Hydroxy-16 $\alpha$-acetoxy-lanosta-7,9(11),24-trien-21-oic acid & {$[12]$} \\
\hline$(53)$ & Polyporenic acid C & {$[17]$} \\
\hline$(54)$ & Dehydrotrametenonic acid & {$[23]$} \\
\hline (55) & $15 \alpha$-Hydroxydehydrotumulosic acid & {$[25]$} \\
\hline$(56)$ & $16 \alpha, 25$-Dihydroxydehydroeburicoic acid & {$[25]$} \\
\hline$(57)$ & 29-Hydroxypolyporenic acid C & {$[16]$} \\
\hline$(58)$ & Poriacosones A & {$[26]$} \\
\hline
\end{tabular}


TABle 1: Continued.

\begin{tabular}{|c|c|c|}
\hline No. & Name & Ref. \\
\hline (59) & Poriacosones B & {$[26]$} \\
\hline$(60)$ & $16 \alpha, 27$-Dihydroxydehydrotrametenoic acid & {$[17]$} \\
\hline$(61)$ & $3 \beta, 16 \alpha, 30$-Trihydroxy-24-methyllanosta-7,9(11),24(31)-trien-21-oic acid & {$[18]$} \\
\hline$(62)$ & $3 \beta$-Acetoxy-16 $\alpha, 24 \beta$-dihydroxylanosta-7,9(11),25-trien-21-oic acid & {$[18]$} \\
\hline$(63)$ & 29-Hydroxydehydrotumulosic acid & {$[27]$} \\
\hline$(64)$ & 29-Hydroxydehydropachymic acid & {$[28]$} \\
\hline$(65)$ & $3 \beta, 15 \alpha$-Dihydroxylanosta-7,9(11),24-triene-21-oic acid & [29] \\
\hline$(66)$ & Dehydrosulphurenic acid & [29] \\
\hline$(67)$ & Dehydroeburicoic acid monoacetate & {$[18]$} \\
\hline$(68)$ & $3 \beta$-Acetoxylanosta-7,9(11),24-trien-21-oic acid & {$[18]$} \\
\hline (69) & Poricoic acid ZE & {$[2]$} \\
\hline$(70)$ & Poricoic acid ZI & {$[2]$} \\
\hline$(71)$ & Poricoic acid ZL & {$[2]$} \\
\hline$(72)$ & Poricoic acid ZV & {$[2]$} \\
\hline$(73)$ & Coriacoic acid B & {$[30]$} \\
\hline (74) & Coriacoic acid C & {$[30]$} \\
\hline$(75)$ & 6,16 $\alpha$-Dihydroxydehydrotrametenonic acid & [16] \\
\hline$(76)$ & $16 \alpha$-Hydroxydehydrotrametenonic acid & [16] \\
\hline$(77)$ & 25,26-Dihydroxydehydropachymic acid & {$[16]$} \\
\hline$(78)$ & $3 \beta, 16 \alpha$-Dihydroxy-24-hydroxymethyllanosta-7,9(11)-dien-21-oic acid & {$[16]$} \\
\hline (79) & $15 \alpha$-Hydroxydehydrotrametenolic acid & [16] \\
\hline$(80)$ & $16 \alpha$-Hydroxydehydrotrametenoic acid & {$[16]$} \\
\hline$(81)$ & 16-Hydroxy-3,24-dioxolanosta-7,9(11)-dien-21-oic acid & {$[16]$} \\
\hline$(82)$ & $16 \alpha$-Acetyloxy-24-methylene-3-oxolanosta-7,9(11)-dien-21-oic acid & {$[16]$} \\
\hline \multicolumn{3}{|c|}{ 3,4-Ring-opening lanoster-8-ene triterpenes (83-93) } \\
\hline$(83)$ & Poricoic acid G & {$[26]$} \\
\hline$(84)$ & Poricoic acid $\mathrm{H}$ & {$[26]$} \\
\hline$(85)$ & 25-Hydroxyporicoic acid $\mathrm{H}$ & {$[25]$} \\
\hline$(86)$ & Poricoic acid GM & [17] \\
\hline$(87)$ & Poricoic acid HM & {$[17]$} \\
\hline$(88)$ & Poricoic acid GE & [29] \\
\hline$(89)$ & Poricoic acid ZA & {$[31]$} \\
\hline$(90)$ & Poricoic acid ZJ & {$[2]$} \\
\hline (91) & Poricoic acid ZK & {$[2]$} \\
\hline$(92)$ & Poricoic acid ZR & {$[21]$} \\
\hline (93) & 25-Methoxy-29-hydroxyporicoic acid HM & {$[16]$} \\
\hline
\end{tabular}

3, 4-Ring-opening lanoster-7,9(11)-diene triterpenes (94-122)

(94)

(96)

(97)

(98)

(99)

(100)

(101)

(102)

(103)

(104)

(105)

(106)

(107)

(108)

(109)

(110)

(111)

(112)

(113)

(114)

(115)

(116)
Poricoic acid A

Poricoic acid B

Poricoic acid C

Poricoic acid D

Poricoic acid DM

Poricoic acid AM

Poricoic acid E

Poricoic acid BM

Poricoic acid $\mathrm{F}$

16-Deoxyporicoic acid B

Poricoic acid CM

25-Methoxyporicoic acid A

26-Hydroxyporicoic acid DM

25-Hydroxyporicoic acid C

Poricoic acid AE

Poricoic acid CE

3,4-Secolanosta-4(28),7,9,24Z-tetraen-3,26-dioic acid

Poricoic acid BE

16 $\alpha$-Hydroxy-3,4-secolanosta-4(28),7,9(11),24(31),25(27)-pentaene-3,21-dioic acid

Poricoic acid ZB

Poricoic acid ZC

Poricoic acid ZD

Poricoic acid ZG
[27]

[27]

[24]

[24]

[24]

[15]

[15]

[15]

[25]

[25]

[17]

[17]

[17]

[32]

[33]

[29]

[2] 
TABle 1: Continued.

\begin{tabular}{llr}
\hline No. & Name & Ref. \\
\hline$(117)$ & Poricoic acid ZM & {$[21]$} \\
$(118)$ & Poricoic acid ZO & {$[21]$} \\
$(119)$ & Poricoic acid ZP & {$[21]$} \\
$(120)$ & Poricoic acid ZN & {$[21]$} \\
$(121)$ & Poricoic acid ZT & {$[2]$} \\
$(122)$ & Poricoic acid ZQ & {$[21]$} \\
\hline
\end{tabular}

Cyclodioxy tetracyclic triterpenes $(123,124)$

$5 \alpha, 8 \alpha$-Peroxydehydrotumulosic acid

(124)

3-(2-Hydroxyacetoxy)- $5 \alpha, 8 \alpha$-peroxydehydrotumulosic acid

4, 5-ring-opening triterpenes $(125,126)$

Daedaleanic acid A

Other tetracyclic triterpenoids (127-131)

(127)

(128)

(129)

(130)

(131)

Pentacyclic triterpenes (132-134)

(132)

(133)

(134)

(3 $\beta, 16 \alpha)$-3-Acetyloxy-16-hydroxy-24-methylenelanosta-5,7(9),11-tetraene-21-oic acid $16 \alpha$-Hydroxy-3-oxo-24-methyllanosta-5,7,9(11),24(31)-tetraen-21-oic acid

6,7-Dehydroporicoic acid $\mathrm{H}$

Coriacoic acid A

Coriacoic acid D

Diterpenes (135-140)

(135)

(136)

(137)

(138)

(139)

(140)

Sterols (141-162)

(141)

(142)

(143)

(144)

(145)

(146)

(147)

(148)

(149)

(150)

(151)

(152)

(153)

(154)

(155)

(156)

(157)

(158)

(159)

(160)

(161)

(162)
$\beta$-Amyrin acetate

[12]

Oleanolic acid

3-O-acetyloleanolic acid

[36]

[1]

7-oxo-15-Hydroxydehydroabietic acid

Dehydroabietic acid methyl ester [37]

Poricoic acid ZF [2]

Dehydroabietic acid $\quad[2]$

7-Oxocallitrisic acid [2] Pimaric acid

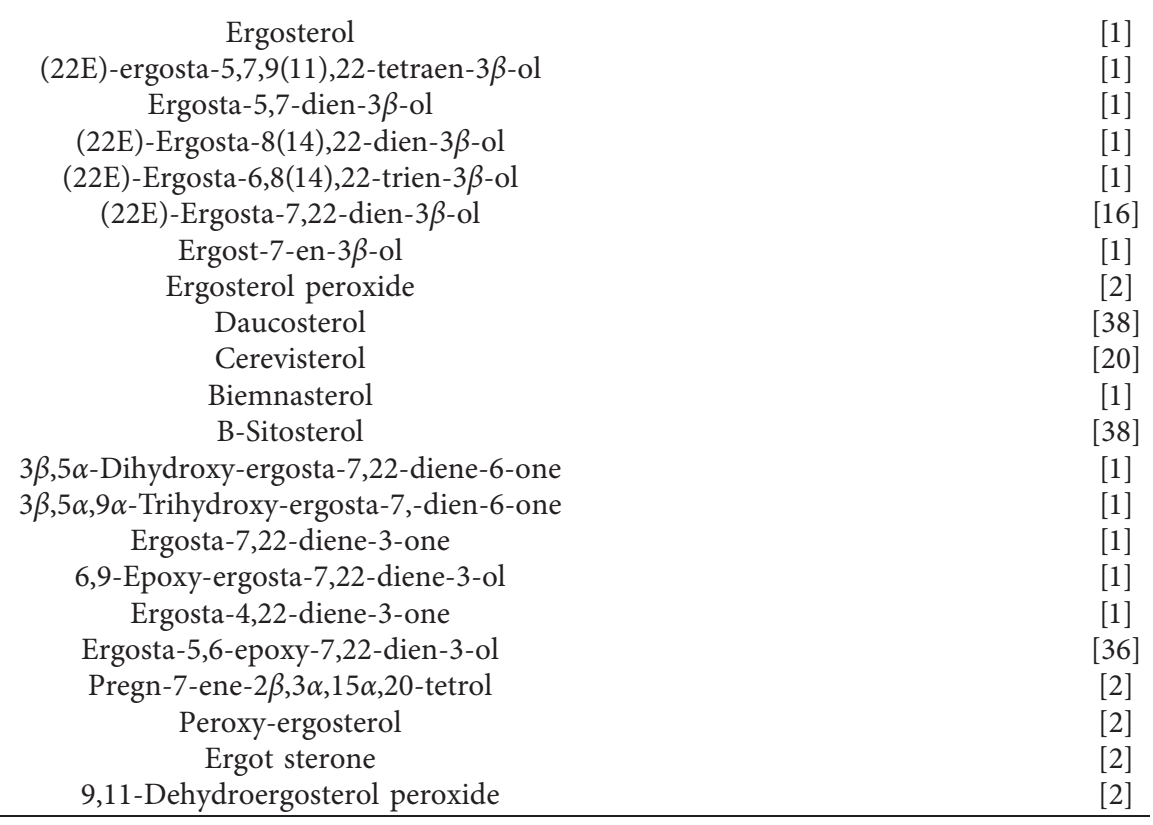




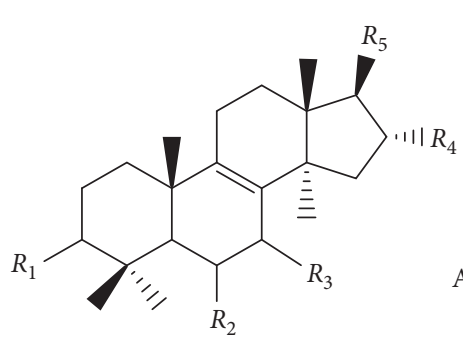

$1 R_{1}=\beta-\mathrm{OAc} R_{2}=\mathrm{H} R_{3}=\mathrm{H} R_{4}=\alpha-\mathrm{OH} R_{5}=\mathrm{a}$

$2 R_{1}=\beta-\mathrm{OH} R_{2}=\mathrm{H} R_{3}=\mathrm{H} R_{4}=\alpha-\mathrm{OH} R_{5}=\mathrm{a}$

$3 R_{1}=\beta-\mathrm{OH} R_{2}=\alpha-\mathrm{H} R_{3}=\mathrm{H} R_{4}=\alpha-\mathrm{H} R_{5}=\mathrm{b}$

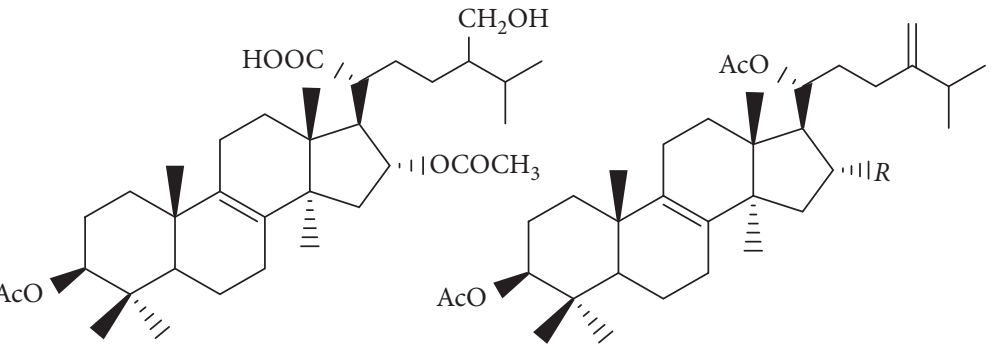

8

$9 R=\mathrm{OAc}$

$10 \mathrm{R}=\mathrm{OH}$

$4 R_{1}=\beta-\mathrm{OH} R_{2}=\mathrm{H} R_{3}=\mathrm{H} R_{4}=\alpha-\mathrm{H} R_{5}=\mathrm{a}$

$5 R_{1}=\beta-\mathrm{OAc} R_{2}=\alpha-\mathrm{H} R_{3}=\mathrm{H} R_{4}=\alpha-\mathrm{OH} R_{5}=\mathrm{b}$

$6 R_{1}=\beta-\mathrm{OH} R_{2}=\alpha-\mathrm{OH} R_{3}=\mathrm{H} R_{4}=\alpha-\mathrm{OH} R_{5}=\mathrm{b}$

$7 R_{1}=\beta-\mathrm{OAc} R_{2}=\mathrm{H} R_{3}=\mathrm{H} R_{4}=\alpha-\mathrm{OAc} R_{5}=\mathrm{a}$

$12 R_{1}=\beta-\mathrm{OAc} R_{2}=\mathrm{H} R_{3}=\mathrm{H} R_{4}=\alpha-\mathrm{OH} R_{5}=\mathrm{e}$

$13 R_{1}=\alpha-\mathrm{OH} R_{2}=\mathrm{H} R_{3}=\mathrm{H} R_{4}=\alpha-\mathrm{OH} R_{5}=\mathrm{e}$

$14 R_{1}==\mathrm{O} R_{2}=\mathrm{H} R_{3}=\mathrm{H} R_{4}=\alpha-\mathrm{OH} R_{5}=\mathrm{e}$

$15 R_{1}==\mathrm{O} R_{2}=\mathrm{H} R_{3}=\mathrm{H} R_{4}=\alpha-\mathrm{OH} R_{5}=\mathrm{e}$

$16 R_{1}=\beta-\mathrm{OH} R_{2}=\mathrm{H} R_{3}==\mathrm{O} R_{4}=\alpha-\mathrm{OH} R_{5}=\mathrm{a}$

$17 R_{1}=\alpha-\mathrm{OH} R_{2}=\mathrm{H} R_{3}==\mathrm{O} R_{4}=\alpha-\mathrm{OH} R_{5}=\mathrm{a}$

$18 R_{1}==\mathrm{O} R_{2}=\mathrm{H} R_{3}=\mathrm{H} R_{4}=\mathrm{H} R_{5}=\mathrm{b}$

$19 R_{1}=\beta-\mathrm{OAc} R_{2}=\mathrm{H} R_{3}=\mathrm{H} R_{4}=\alpha-\mathrm{H} R_{5}=\mathrm{a}$<smiles></smiles>

11

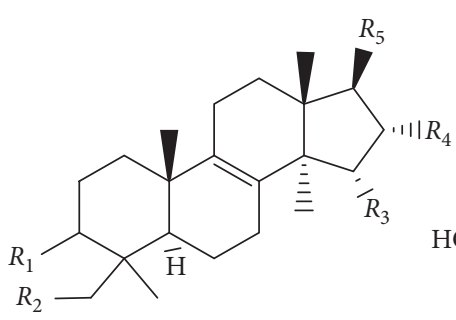

$23 R_{1}=\beta-\mathrm{OH} R_{2}=\mathrm{H} R_{3}=\mathrm{OH} R_{4}=\mathrm{H} R_{5}=\mathrm{c}^{\prime}$

$24 R_{1}=\beta-\mathrm{OAc} R_{2}=\mathrm{H} R_{3}=\mathrm{H} R_{4}=\mathrm{OH} R_{5}=\mathrm{c}^{\prime}$

$25 R_{1}=\alpha-\mathrm{OH} R_{2}=\mathrm{H} R_{3}=\mathrm{H} R_{4}=\mathrm{OH} R_{5}=\mathrm{c}^{\prime}$

$26 R_{1}==\mathrm{O} R_{2}=\mathrm{H} R_{3}=\mathrm{OH} R_{4}=\mathrm{H} R_{5}=\mathrm{c}^{\prime}$

$27 R_{1}=\alpha-\mathrm{OH} R_{2}=\mathrm{H} R_{3}=\mathrm{H} R_{4}=\mathrm{OH} R_{5}=\mathrm{d}^{\prime}$

$28 R_{1}==\mathrm{O} R_{2}=\mathrm{OH} R_{3}=\mathrm{H} R_{4}=\mathrm{OH} R_{5}=\mathrm{a}^{\prime}$

$29 R_{1}=\beta-\mathrm{OAc} R_{2}=\mathrm{H} R_{3}=\mathrm{H} R_{4}=\mathrm{OH} R_{5}=\mathrm{d}^{\prime}$

$30 R_{1}==\mathrm{O} R_{2}=\mathrm{H} R_{3}=\mathrm{OH} R_{4}=\mathrm{H} R_{5}=\mathrm{b}^{\prime}$

$31 R_{1}==\mathrm{O} R_{2}=\mathrm{H} R_{3}=\mathrm{H} R_{4}=\mathrm{OH} R_{5}=\mathrm{b}^{\prime}$

$32 R_{1}=\beta-\mathrm{OAc} R_{2}=\mathrm{OH} R_{3}=\mathrm{H} R_{4}=\mathrm{OAc} R_{5}=\mathrm{b}^{\prime}$

$33 R_{1}=\beta-\mathrm{OH} R_{2}=\mathrm{OH} R_{3}=\mathrm{H} R_{4}=\mathrm{H} R_{5}=\mathrm{a}^{\prime}$

$34 R_{1}=\beta-\mathrm{OAc} R_{2}=\mathrm{H} R_{3}=\mathrm{OAc} R_{4}=\mathrm{H} R_{5}=\mathrm{a}^{\prime}$

$35 R_{1}==\mathrm{O} R_{2}=\mathrm{H} R_{3}=\mathrm{H} R_{4}=\mathrm{OAc} R_{5}=\mathrm{a}^{\prime}$

$36 R_{1}=\alpha-\mathrm{OAc} R_{2}=\mathrm{H} R_{3}=\mathrm{H} R_{4}=\mathrm{OH} R_{5}=\mathrm{a}^{\prime}$<smiles>C=C(CCC(C(=O)O)[C@H]1[C@H](O)C[C@]2(C)C3=C(CC[C@]12C)[C@@]1(C)CCCC(C)(C)C1=CC3=O)C(C)C</smiles>

20

21

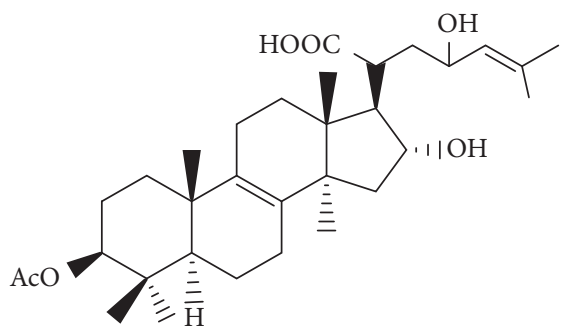

(a)

FIgUre 1: Continued. 


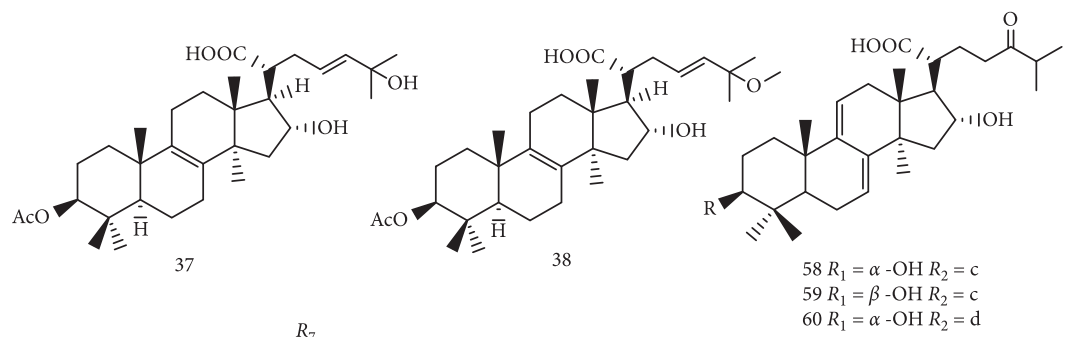

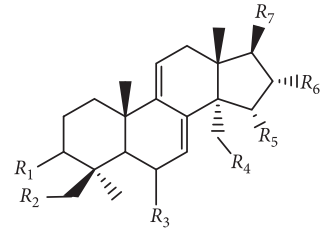

$39 R_{1}=\beta-\mathrm{OH} R_{2}=\mathrm{H} R_{3}=\mathrm{H} R_{4}=\mathrm{H} R_{5}=\alpha-\mathrm{H} R_{6}=\alpha-\mathrm{H} R_{7}=\mathrm{b}^{\prime}$ $40 R_{1}=\beta-\mathrm{OAc} R_{2}=\mathrm{H} R_{3}=\alpha-\mathrm{H} R_{4}=\mathrm{H} R_{5}=\mathrm{H} R_{6}=\alpha-\mathrm{OH} R_{7}=\mathrm{a}$ $41 R_{1}=\beta-\mathrm{OH} R_{2}=\mathrm{H} R_{3}=\alpha-\mathrm{H} R_{4}=\mathrm{H} R_{5}=\mathrm{H} R_{6}=\alpha-\mathrm{H} R_{7}=\mathrm{a}$ $42 R_{1}==\mathrm{O} R_{2}=\mathrm{H} R_{3}=\alpha-\mathrm{OH} R_{4}=\mathrm{H} R_{5}=\mathrm{H} R_{6}=\alpha-\mathrm{OH} R_{7}=\mathrm{e}^{\prime}$ $43 R_{1}=\alpha-\mathrm{OH} R_{2}=\mathrm{H} R_{3}=\mathrm{H} R_{4}=\mathrm{H} R_{5}=\mathrm{H} R_{6}=\alpha-\mathrm{OH} R_{7}=\mathrm{a}$ $44 R_{1}=\alpha-\mathrm{OH} R_{2}=\mathrm{H} R_{3}=\mathrm{H} R_{4}=\mathrm{H} R_{5}=\mathrm{H} R_{6}=\alpha-\mathrm{OH} R_{7}=\mathrm{e}$ $45 R_{1}=\beta-\mathrm{OH} R_{2}=\mathrm{H} \mathrm{R}_{3}=\alpha-\mathrm{H} \mathrm{R}_{4}=\mathrm{H} \mathrm{R}_{5}=\mathrm{H} R_{6}=\alpha-\mathrm{H} R_{7}=\mathrm{a}$ $46 R_{1}==\mathrm{O} R_{2}=\mathrm{H} R_{3}=\alpha-\mathrm{H} R_{4}=\mathrm{H} R_{5}=\mathrm{H} R_{6}=\alpha-\mathrm{H} R_{7}=\mathrm{a}^{4}$ $47 R_{1}=\beta-O R_{2} R_{2}=H R_{3}=H R_{4}=H R_{5}=\alpha-H R_{6}=\alpha-O H R_{7}=b^{\prime}$ $47 R_{1}=\beta-O A R_{2}=H R_{3}-H R_{4}=H R_{5}=\alpha R_{6}=a-H R_{7}=a^{\prime}$ $48 R_{1}=\alpha-O A C R_{2}=H R_{3}=H R_{4}=H R_{5}=H R_{6}=\alpha-O H R_{7}=\mathrm{a}$ $49 R_{1}=\beta-\mathrm{OH} R_{2}=\mathrm{H} R_{3}=\mathrm{H} \mathrm{R}_{4}=\mathrm{H} R_{5}=\alpha-\mathrm{H} R_{6}=\alpha-\mathrm{OH} R_{7}=\mathrm{b}^{\prime}$ $50 R_{1}=\beta-\mathrm{OAc} R_{2}=\mathrm{H} \mathrm{R}_{3}=\alpha-\mathrm{OH} R_{4}=\mathrm{H} R_{5}=\mathrm{H} R_{6}=\alpha-\mathrm{OH} R_{7}=\mathrm{a}$ $51 R_{1}=\beta$-p-hydroxybenzoyl $R_{2}=\mathrm{H} R_{3}=\alpha-\mathrm{H} R_{4}=\mathrm{H} R_{5}=\mathrm{H} R_{6}=\alpha-\mathrm{OH} R_{7}=\mathrm{a}$ $52 R_{1}=\beta-\mathrm{OH} R_{2}=\mathrm{H} R_{3}=\mathrm{H} R_{4}=\mathrm{H} R_{5}=\alpha-\mathrm{H} R_{6}=\alpha-\mathrm{OAc} R_{7}=\mathrm{b}$ $53 R_{1}==\mathrm{O}_{2}=\mathrm{H} R_{3}=\alpha-\mathrm{OH} \mathrm{R}_{4}=\mathrm{HR}_{5}=\mathrm{HR}_{6}=\alpha-\mathrm{OH} R_{7}=\mathrm{e}^{\prime}$ $54 R_{1}==\mathrm{O} R_{2}=\mathrm{H} R_{3}=\mathrm{H} R_{4}=\mathrm{H} R_{5}=\mathrm{H} R_{6}=\mathrm{H} R_{7}=\mathrm{b}$

$55 R_{1}=\beta-\mathrm{OH} R_{2}=\mathrm{H} R_{3}=\mathrm{H} R_{4}=\mathrm{H} R_{5}=\alpha-\mathrm{OH} R_{6}=\alpha-\mathrm{OH} R_{7}=\mathrm{a}$ $56 R_{1}==\mathrm{O} R_{2}=\mathrm{H} R_{3}=\alpha-\mathrm{H} R_{4}=\mathrm{H} R_{5}=\mathrm{H} R_{6}=\alpha-\mathrm{OH} R_{7}=\mathrm{e}^{\prime}$ $57 R_{1}==\mathrm{O} R_{2}=\mathrm{OH} R_{3}=\alpha-\mathrm{H} R_{4}=\mathrm{H} R_{5}=\mathrm{H} R_{6}=\alpha-\mathrm{OH} R_{7}=\mathrm{a}^{\prime}$ $61 R_{1}=\alpha-\mathrm{OH} R_{2}=\mathrm{H} R_{3}=\mathrm{H} R_{4}=\mathrm{OH} R_{5}=\mathrm{H} R_{6}=\alpha-\mathrm{OH} R_{7}=\mathrm{a}$ $63 R_{\mathrm{C}^{-}} \mathrm{OH} R_{2}=\mathrm{OH} R_{3}=\mathrm{H} R_{4}=\mathrm{H} R_{5}=\mathrm{H} R_{6}=\alpha-\mathrm{OH} R_{7}=\mathrm{a}$ $64 R_{1}=\alpha-\mathrm{OAc} R_{2}=\mathrm{OH} R_{3}=\mathrm{H} R_{4}=\mathrm{H} R_{5}=\mathrm{H} R_{6}=\alpha-\mathrm{OH} R_{7}=\mathrm{a}$ $65 R_{1}=\beta-\mathrm{OH} R_{2}=\mathrm{H} R_{3}=\mathrm{H} R_{4}=\mathrm{H} R_{5}=\alpha-\mathrm{OH} R_{6}=\alpha-\mathrm{H} R_{7}=\mathrm{a}^{\prime}$ $66 R_{1}=\beta-\mathrm{OH} R_{2}=\mathrm{H} R_{3}=\mathrm{H} R_{4}=\mathrm{H} R_{5}=\alpha-\mathrm{OH} R_{6}=\alpha-\mathrm{H} R_{7}=\mathrm{b}^{\prime}$ $67 R_{1}=\alpha-\mathrm{OAc} R_{2}=\mathrm{H} R_{3}=\mathrm{H} R_{4}=\mathrm{H} R_{5}=\mathrm{H} R_{6}=\alpha-\mathrm{H} R_{7}=\mathrm{a}$ $68 R_{1}=\beta-\mathrm{OAc} R_{2}=\mathrm{H} R_{3}=\mathrm{H} R_{4}=\mathrm{H} R_{5}=\alpha-\mathrm{H} R_{6}=\alpha-\mathrm{H} R_{7}=\mathrm{b}$ $70 R_{1}==\mathrm{O} R_{2}=\mathrm{H} R_{3}=\beta-\mathrm{OH} R_{4}=\mathrm{H} R_{5}=\mathrm{H} R_{6}=\alpha-\mathrm{OH} R_{7}=\mathrm{c}^{\prime}$

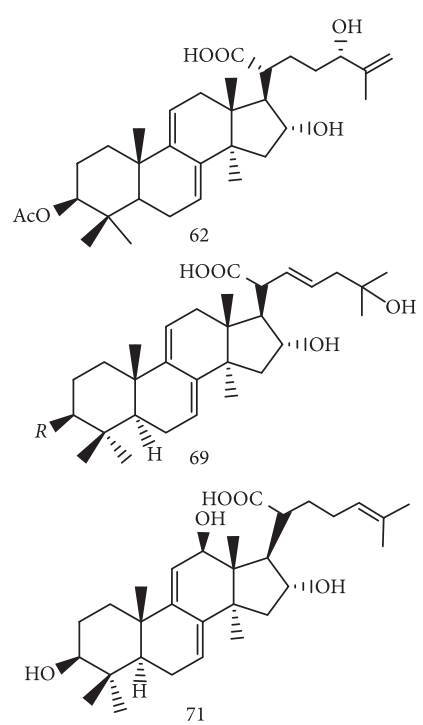

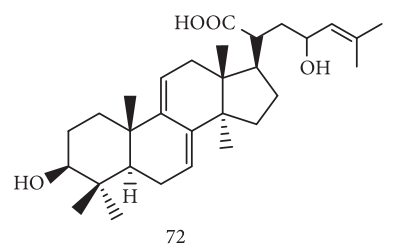

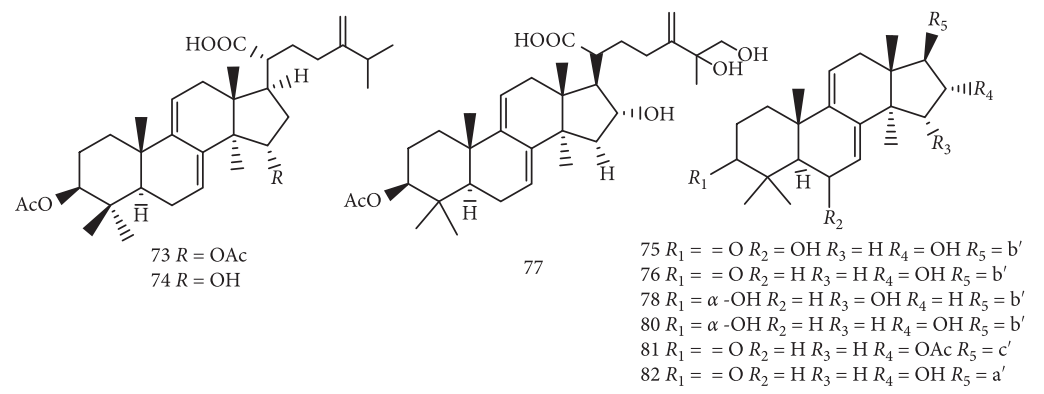

(b)

Figure 1: Continued. 


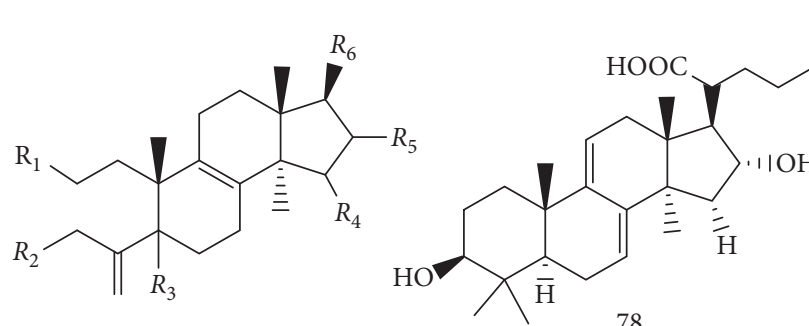

$83 R_{1}=\mathrm{COOH} R_{2}=\mathrm{H} R_{3}=\mathrm{H} R_{4}=\mathrm{H} R_{5}=\alpha-\mathrm{OH} R_{6}=\mathrm{b}^{\prime}$

$84 R_{1}=\mathrm{COOH} R_{2}=\mathrm{H} R_{3}=\mathrm{H} R_{4}=\mathrm{H} R_{5}=\alpha-\mathrm{OH} R_{6}=\mathrm{a}$

$85 R_{1}=\mathrm{COOH} R_{2}=\mathrm{H} R_{3}=\mathrm{H} R_{4}=\mathrm{H} R_{5}=\alpha-\mathrm{OH} R_{6}=\mathrm{e}$

$86 R_{1}=\mathrm{COOCH}_{3} R_{2}=\mathrm{H} R_{3}=\mathrm{H} R_{4}=\mathrm{H} R_{5}=\alpha-\mathrm{OH} R_{6}=\mathrm{b}^{\prime}$

$87 R_{1}=\mathrm{COOCH}_{3} R_{2}=\mathrm{H} R_{3}=\mathrm{H} R_{4}=\mathrm{H} R_{5}=\alpha-\mathrm{OH} R_{6}=\mathrm{a}$

$88 R_{1}=\mathrm{COOCH}_{2} \mathrm{CH}_{3} R_{2}=\mathrm{H} R_{3}=\mathrm{H} R_{4}=\mathrm{H} R_{5}=\alpha-\mathrm{OH} R_{6}=\mathrm{b}^{\prime}$

$89 R_{1}=\mathrm{COOH} R_{2}=\mathrm{H} \mathrm{R}_{3}=\mathrm{H} R_{4}=\mathrm{H} R_{5}=\alpha-\mathrm{OH} R_{6}=\mathrm{d}$

$90 R_{1}=\mathrm{COOH} R_{2}=\mathrm{H} \mathrm{R}_{3}=\alpha-\mathrm{H} R_{4}=\alpha-\mathrm{OH} R_{5}=\mathrm{H} R_{6}=\mathrm{a}^{\prime}$

$91 R_{1}=\mathrm{COOCH}_{3} R_{2}=\mathrm{H} \mathrm{R}_{3}=\alpha-\mathrm{H} R_{4}=\mathrm{H} R_{5}=\alpha-\mathrm{OH} R_{6}=\mathrm{b}^{\prime}$

$92 R_{1}=\mathrm{COOCH}_{3} R_{2}=\mathrm{H} \mathrm{R}_{3}=\alpha-\mathrm{H} R_{4}=\mathrm{H} R_{5}=\alpha-\mathrm{OH} R_{6}=\mathrm{d}^{\prime}$<smiles>[R4]CC[C@]1(C)C2=CC[C@]3(C)[C@@H]([R6])C([R5])[C@@H]([R])[C@@]3(C)C2=CC[C@@]1([R3])C(=C)C[R4]</smiles>

$94 R_{1}=\mathrm{COOH} R_{2}=\mathrm{H} R_{3}=\mathrm{H} R_{4}=\mathrm{H} R_{5}=\alpha-\mathrm{OH} R_{6}=\mathrm{a}^{\prime}$

$95 R_{1}=\mathrm{COOH} R_{2}=\mathrm{H} R_{3}=\mathrm{H} R_{4}=\mathrm{H} R 5=\alpha-\mathrm{OH} R_{6}=\mathrm{b}^{\prime}$

$96 R_{1}=\mathrm{COOH} R_{2}=\mathrm{H} R_{3}=\mathrm{H} R_{4}=\mathrm{H} R_{5}=\alpha-\mathrm{H} R_{6}=\mathrm{a}^{\prime}$

$97 R_{1}=\mathrm{COOH} R_{2}=\mathrm{H} R_{3}=\mathrm{H} R_{4}=\mathrm{H} R_{5}=\alpha-\mathrm{OH} R_{6}=\mathrm{b}^{\prime}$

$98 R_{1}=\mathrm{COOCH}_{3} R_{2}=\mathrm{H} R_{3}=\mathrm{H} R_{4}=\mathrm{H} R_{5}=\alpha-\mathrm{OH} R_{6}=\mathrm{b}^{\prime}$

$99 R_{1}=\mathrm{COOCH}_{3} R_{2}=\mathrm{H} R_{3}=\mathrm{H} R_{4}=\mathrm{H} R_{5}=\alpha-\mathrm{OH} R_{6}=\mathrm{a}^{\prime}$

$100 R_{1}=\mathrm{COOH} R_{2}=\mathrm{H} R_{3}=\mathrm{H} R_{4}=\mathrm{H} R_{5}=\alpha-\mathrm{OH} R_{6}=\mathrm{d}^{\prime}$

$101 R_{1}=\mathrm{COOCH}_{3} R_{2}=\mathrm{H} R_{3}=\mathrm{H} R_{4}=\mathrm{H} R_{5}=\alpha-\mathrm{OH} R_{6}=\mathrm{b}^{\prime}$

$102 R_{1}=\mathrm{COOH} R_{2}=\mathrm{OH} R_{3}=\mathrm{H} R_{4}=\mathrm{H} R_{5}=\alpha-\mathrm{OH} R_{6}=\mathrm{a}^{\prime}$

$103 R_{1}=\mathrm{COOH} R_{2}=\mathrm{H} R_{3}=\mathrm{H} R_{4}=\mathrm{H} R_{5}=\alpha-\mathrm{H} R_{6}=\mathrm{b}^{\prime}$

$104 R_{1}=\mathrm{COOCH}_{3} \mathrm{R}_{2}=\mathrm{H} \mathrm{R}_{3}=\mathrm{H} \mathrm{R}_{4}=\mathrm{H} R_{5}=\alpha-\mathrm{H} R_{6}=\mathrm{a}^{\prime}$

$107 R_{1}=\mathrm{COOH} R_{2}=\mathrm{H} R_{3}=\mathrm{H} R_{4}=\mathrm{H} R_{5}=\alpha-\mathrm{H} R_{6}=\mathrm{b}^{\prime}$

$108 R_{1}=\mathrm{COOCH}_{2} \mathrm{CH}_{3} R_{2}=\mathrm{H} R_{3}=\mathrm{H} R_{4}=\mathrm{H} R_{5}=\alpha-\mathrm{OH} R_{6}=\mathrm{a}^{\prime}$

$109 R_{1}=\mathrm{COOCH}_{2} \mathrm{CH}_{3} R_{2}=\mathrm{H} R_{3}=\mathrm{H} R_{4}=\mathrm{H} R_{5}=\alpha-\mathrm{H} R_{6}=\mathrm{a}^{\prime}$

$111 R_{1}=\mathrm{COOCH}_{2} \mathrm{CH} 3 R_{2}=\mathrm{H} R_{3}=\mathrm{H} R_{4}=\mathrm{H} R_{5}=\alpha-\mathrm{OH} R_{6}=\mathrm{b}^{\prime}$

$114 R_{1}=\mathrm{COOH} R_{2}=\mathrm{OH} R_{3}=\alpha-\mathrm{H} R_{4}=\mathrm{H} R_{5}=\alpha-\mathrm{OH} R_{6}=\mathrm{b}^{\prime}$

$119 R_{1}=\mathrm{COOCH}_{3} R_{2}=\mathrm{OH} R_{3}=\alpha-\mathrm{H} R_{4}=\mathrm{H} R_{5}=\alpha-\mathrm{OH} R_{6}=\mathrm{a}^{\prime}$

$120 R_{1}=\mathrm{COOH} R_{2}=\mathrm{H} R_{3}=\alpha-\mathrm{H} R_{4}=\alpha-\mathrm{H} R_{5}=\mathrm{H} R_{6}=\mathrm{a}^{\prime}$

$122 R_{1}=\mathrm{COOCH}_{3} R_{2}=\mathrm{OH} R_{3}=\alpha-\mathrm{H}_{4}=\mathrm{H} \mathrm{R}_{5}=\alpha-\mathrm{OH} R_{6}=\mathrm{a}^{\prime}$<smiles>C=C(C)C1CC=C2C(=CC[C@]3(C)[C@@H](C(=C)CCC(=C)C(C)(C)OC)[C@@H](O)C[C@]23C)[C@]1(C)CCC(=O)O</smiles>

105<smiles>C=C(C)C1CC=C2C(=CC[C@@]3(C)[C@@H](C(=O)O)[C@H](O)C[C@]23C)[C@]1(C)CCC(=O)C(C)(O)CO</smiles>

106<smiles>C=C(C)C1CC=C2C3=CC[C@@H]([C@H](C)CCC=C(C)C(=O)O)[C@]3(C)CC=C2[C@]1(C)CCC(=O)O</smiles><smiles>C=C(CC[C@H](C)[C@H]1[C@H](O)C[C@@]2(C)C3=CCC(C(=C)C)[C@@](C)(CCC(=O)O)C3=CC[C@]12C)C(C)C</smiles>

(c)

Figure 1: Continued. 


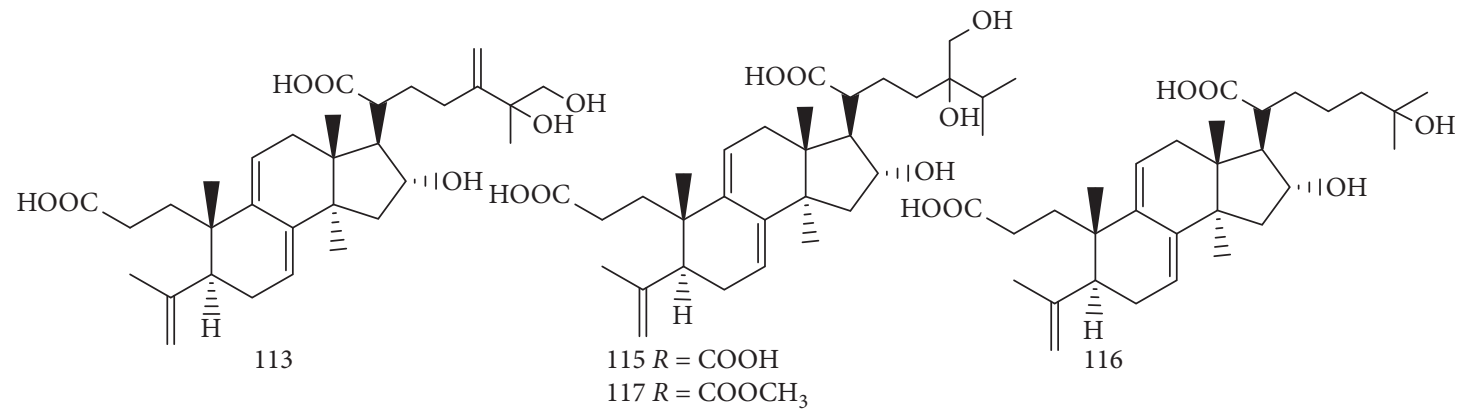

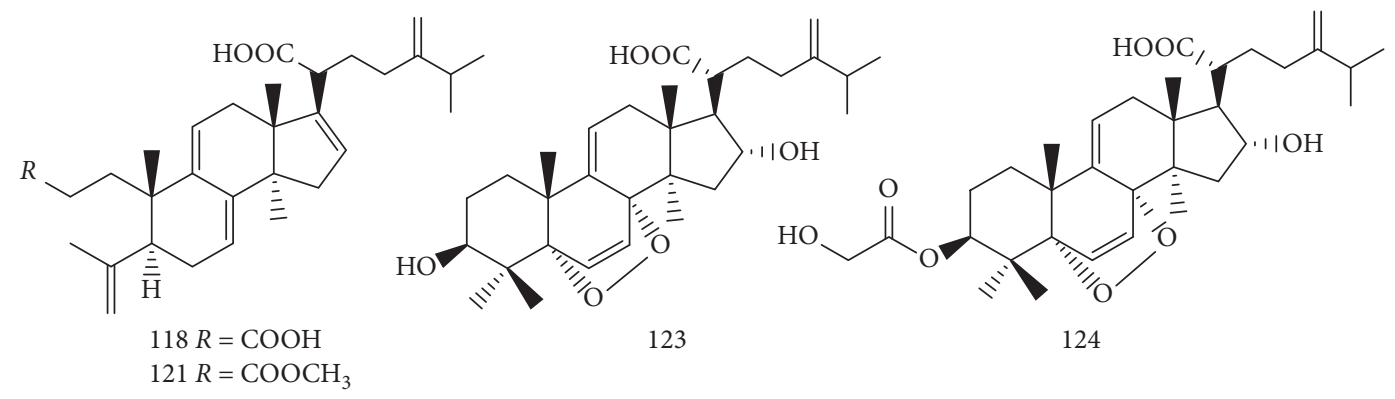

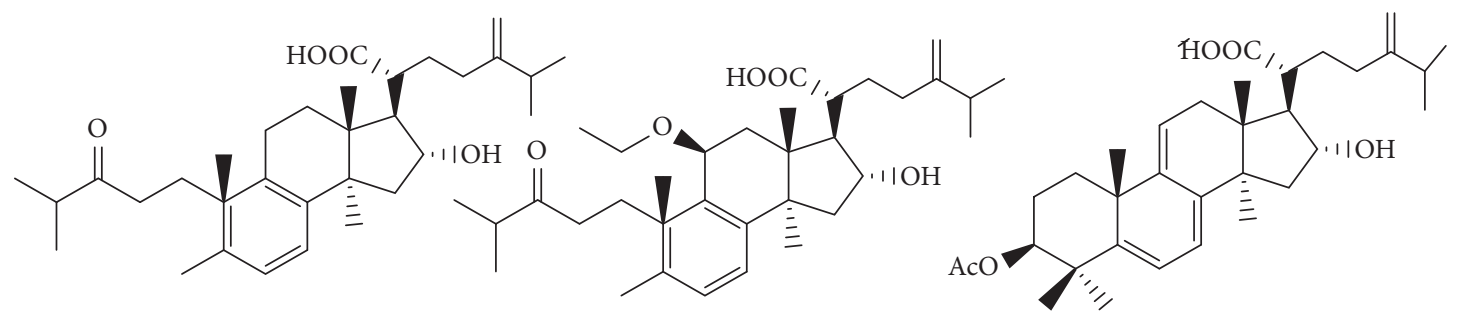

125

126

127

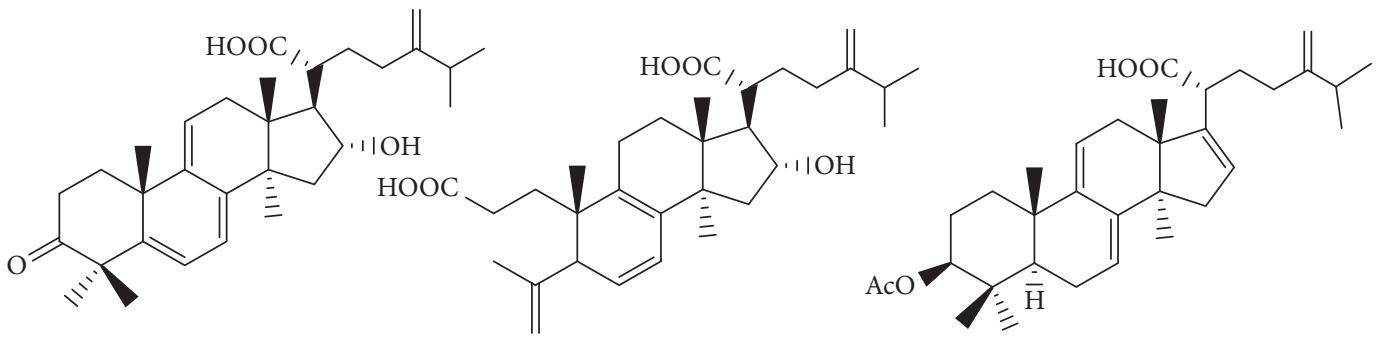

128

129

(d)

FIgURE 1: Continued. 


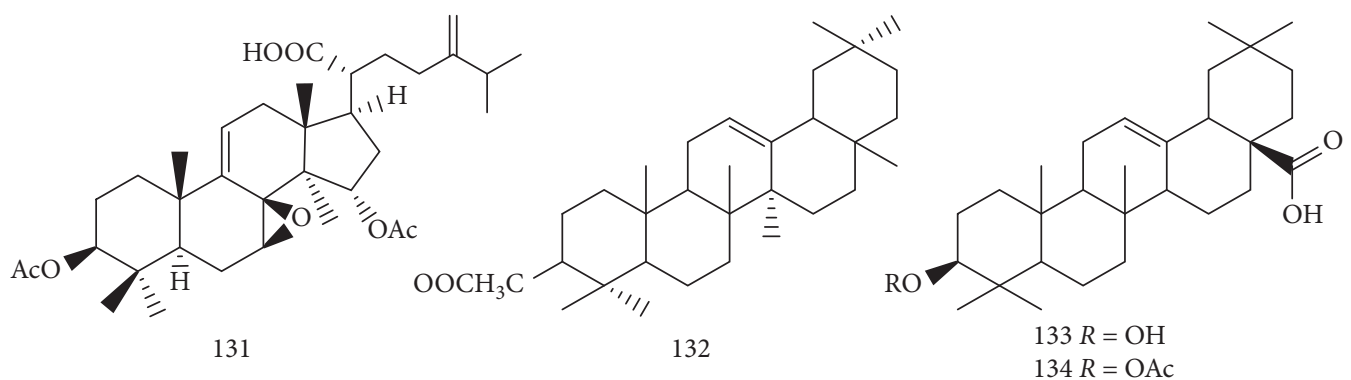<smiles>CC(C)(O)c1ccc2c(c1)C(=O)C[C@@H]1[C@@](C)(C(=O)O)CCC[C@]21C</smiles>

135<smiles>C=C[C@]1(C)C=C2C(=O)CC3[C@@](C)(C(=O)O)CCC[C@]3(C)C2CC1</smiles>

140

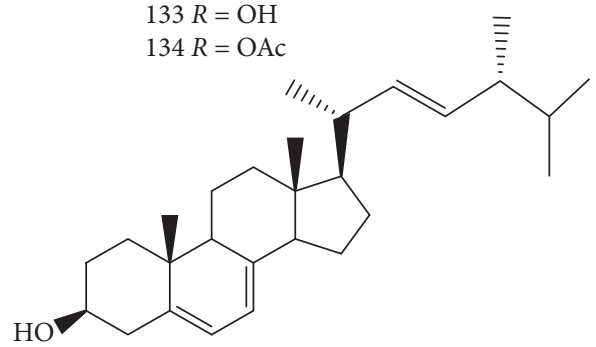

141<smiles>[R]C1C[C@@H]2[C@@H](C)CC([R])c3cc(C(C)C)ccc3[C@@]2(C)C1</smiles><smiles>CC[C@H](C)[C@@H]1C[C@H]2C1CC1C3=CC=C4C[C@@H](O)CC[C@]4(C)C3=CC[C@@]12C</smiles>

142

143

$136 R_{1}=\alpha-\mathrm{COOCH}_{3} R_{2}=\mathrm{H} R_{3}=\mathrm{H}$

$137 R_{1}=\mathrm{H} R_{2}=\mathrm{O}\left(\mathrm{CH}_{2}\right)_{7} \mathrm{CH}_{3} R_{3}=\mathrm{COOH}$ $138 R_{1}=\alpha-\mathrm{COOH} R_{2}=R_{3}=\mathrm{H}$

$139 R_{1}=\alpha-\mathrm{COOH} R_{2}==\mathrm{O} R_{3}=\mathrm{H}$

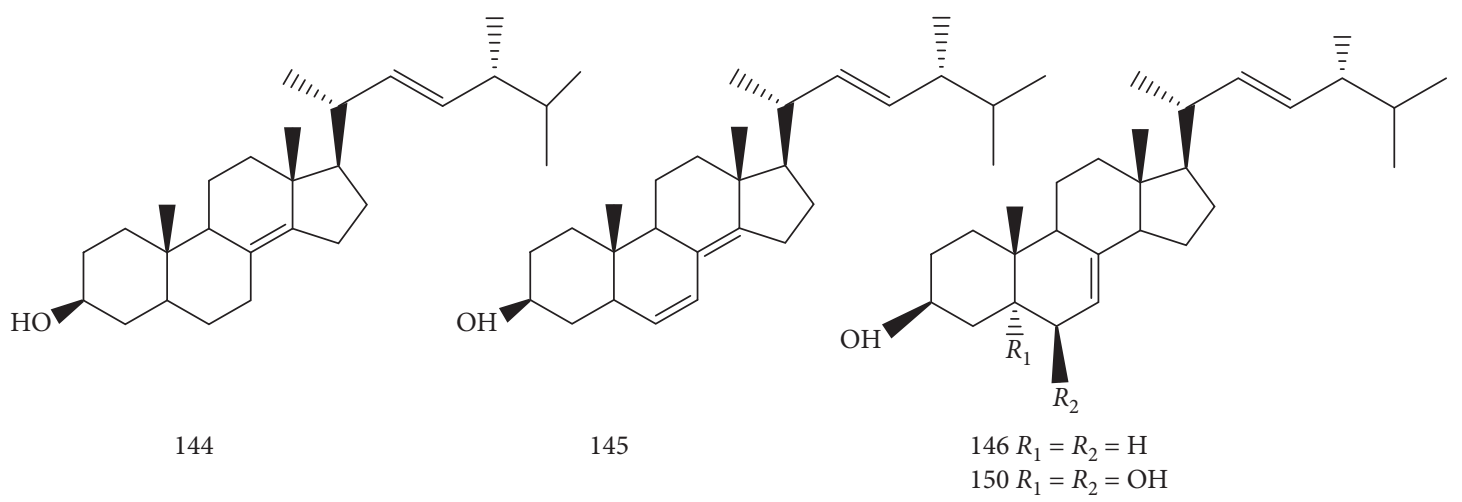

(e)

Figure 1: Continued. 

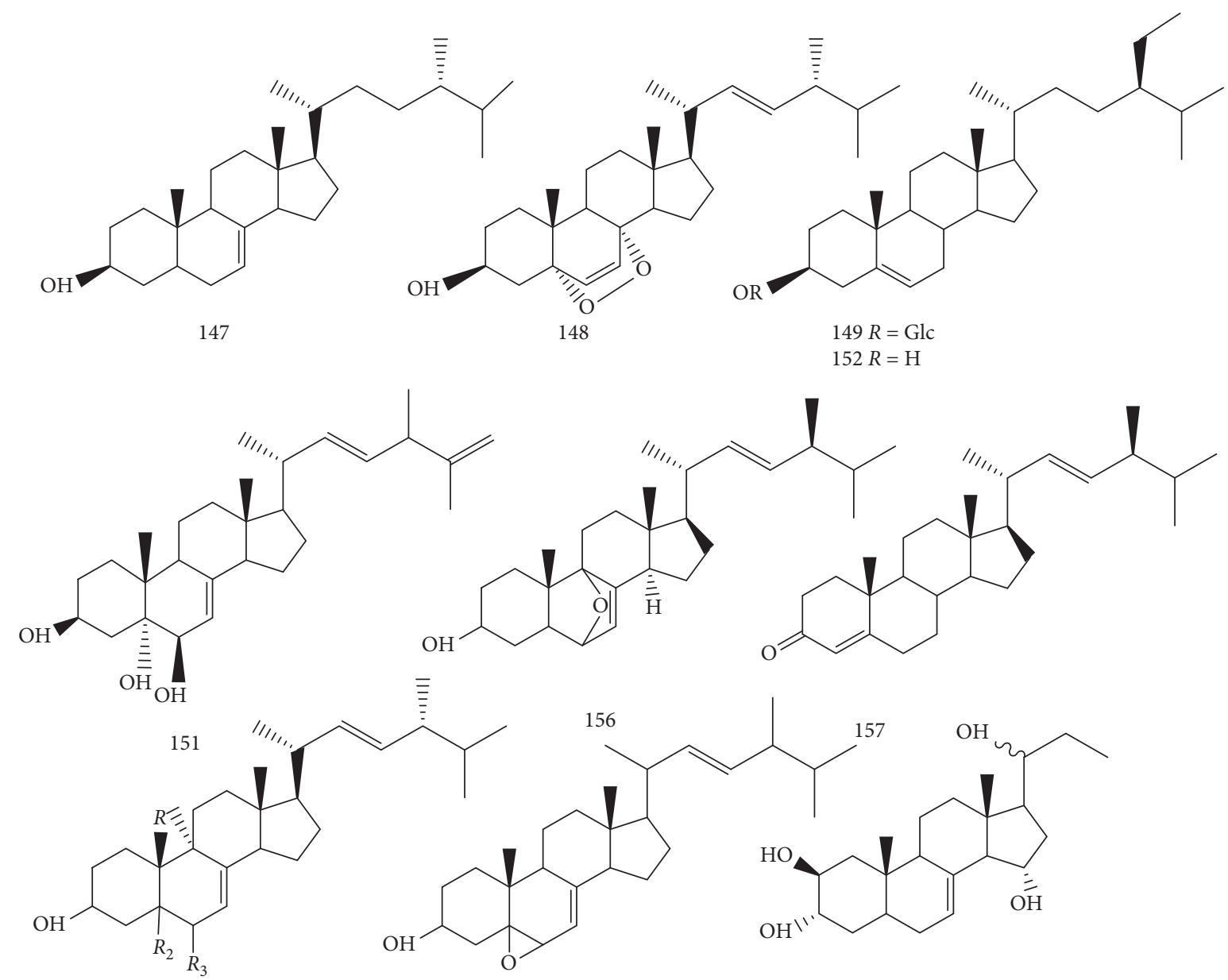

$153 R_{1}=\beta-\mathrm{OH} R_{2}=\alpha-\mathrm{OH} R_{3}==\mathrm{O}$

$154 R_{1}=\beta-\mathrm{OH} R_{2}=\alpha-\mathrm{OH} R_{3}==\mathrm{O}$

$155 R_{1}==\mathrm{O} R_{2}=\mathrm{H} R_{3}=\mathrm{H}$

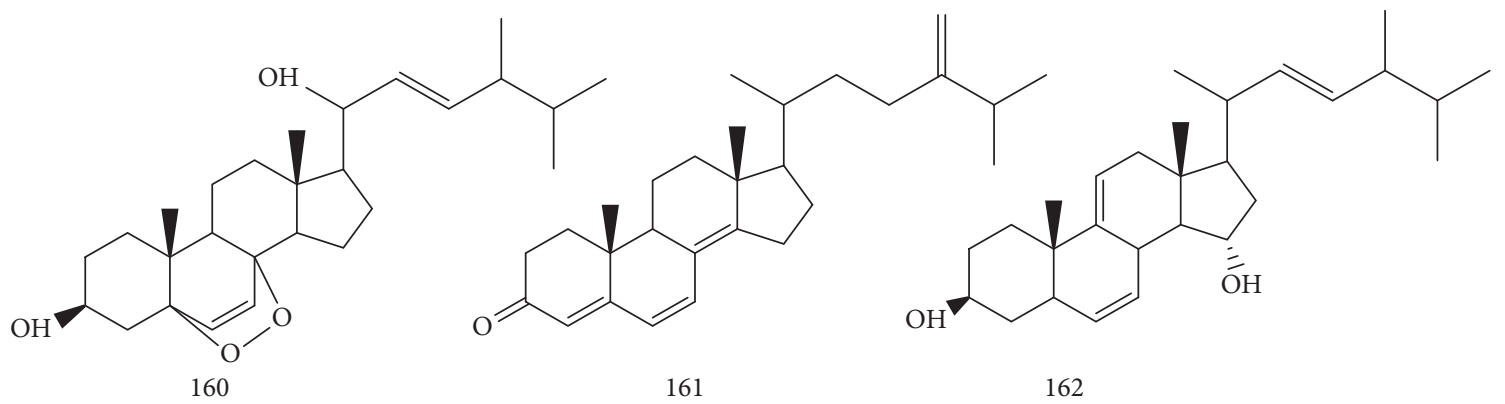<smiles>C=C(CC[C@@H](C)C(=O)O)C(C)C</smiles><smiles>C/C(=C/CCC(=O)O)CO</smiles>

HOOC<smiles>C=C(CCC)C(C)C</smiles><smiles>CC(C)=CCC[C@H](C)C(=O)O</smiles>

$\mathrm{b}^{\prime}$<smiles>CC(C)C(=O)CC[C@H](C)C(=O)O</smiles><smiles>C/C(=C/CC[C@H](C)C(=O)O)CO</smiles><smiles>C=C(CC[C@@H](C)C(=O)O)C(C)(C)CC[C@H](C)C(=O)O</smiles>

(f) 
TABle 2: Polysaccharides isolated from Poria.

\begin{tabular}{|c|c|c|}
\hline No. & Name & Ref. \\
\hline (1) & Pachyman & [39] \\
\hline (2) & PolysaccharideH11 & {$[40]$} \\
\hline (3) & $\mathrm{PC} 1, \mathrm{PC} 2, \mathrm{PC} 2-\mathrm{A}$ & {$[40]$} \\
\hline$(4)$ & PC3 & {$[40]$} \\
\hline (5) & PC4 & {$[41]$} \\
\hline (6) & PCSC22 & {$[24]$} \\
\hline (7) & PCM1, PCM2 & {$[24]$} \\
\hline$(8)$ & РCM3 & {$[24]$} \\
\hline (9) & PCM4 & {$[42]$} \\
\hline$(10)$ & ac-PCM0, ac-PCM1, ac-PCM2, PCPWP, PCPWPS & {$[42]$} \\
\hline$(11)$ & ac-PCM3-I, ac-PCM3-II & {$[42]$} \\
\hline$(12)$ & ac-PCM4-I, ac-PCM4-II & {$[43]$} \\
\hline (13) & wb-PCM1, wb-PCM2, wc-PCM1, wc-PCM2 & {$[42]$} \\
\hline (14) & $\begin{array}{c}\text { wb-PCM3-I, wc-PCM3-I, wb-PCM3-II, wb-PCM4-I, } \\
\text { wb }\end{array}$ & [21] \\
\hline$(15)$ & $\begin{array}{l}\text { PCM4-II, wc-PCM3-II, wc-PCM4I, wc-PCM4-II, } \\
\text { WIP }\end{array}$ & {$[43]$} \\
\hline$(16)$ & wb-PCM0, wc-PCM0 & {$[44]$} \\
\hline$(17)$ & $\begin{array}{c}\text { ab-PCM0, ab-PCM1, ab-PCM2-I, ab-PCM2-I, ab- } \\
\text { PCM3-I }\end{array}$ & {$[44]$} \\
\hline$(18)$ & ab-PCM3-II, ab-PCM4-I, abPCM4-II & {$[45]$} \\
\hline (19) & PCS1, PCS2, PCS3-I & {$[45]$} \\
\hline$(20)$ & PCS3-II & {$[45]$} \\
\hline$(21)$ & PCS4-I & {$[45]$} \\
\hline$(22)$ & PCS4-II & {$[46]$} \\
\hline (23) & PC-PS & {$[24]$} \\
\hline$(24)$ & PCSG & {$[47]$} \\
\hline$(25)$ & Pi-PCM0, Pi-PCM1, Pi-PCM2 & {$[47]$} \\
\hline$(26)$ & Pi-PCM3-I, Pi-PCM4-I & {$[47]$} \\
\hline$(27)$ & Pi-PCM3-II & {$[47]$} \\
\hline$(28)$ & Pi-PCM4-II & {$[48]$} \\
\hline (29) & Polysaccharides from Poria & {$[24]$} \\
\hline (30) & WSP, WSP-1, WSP-2 & {$[24]$} \\
\hline$(31)$ & PCII & [24] \\
\hline (32) & PCP & [49] \\
\hline (33) & ATPCP & {$[50]$} \\
\hline (34) & PCP-II & {$[24]$} \\
\hline (35) & CMP33 & {$[51]$} \\
\hline (36) & PAC & {$[52]$} \\
\hline (37) & FMGP & {$[53]$} \\
\hline (38) & CMP3 & {$[54]$} \\
\hline (39) & PPS, CMP & {$[55]$} \\
\hline$(40)$ & PPSW-1, Sul-W-1 & {$[56]$} \\
\hline$(41)$ & S-CMP & {$[57]$} \\
\hline$(42)$ & Polysaccharides (WRP) & {$[58]$} \\
\hline (43) & $\mathrm{PPC}$ & [59] \\
\hline$(44)$ & PCP-M & {$[60]$} \\
\hline$(45)$ & Sulfated pachymaran & {$[61]$} \\
\hline (46) & PCWPW and PCWPS & {$[62]$} \\
\hline
\end{tabular}

indicated that S-CMP had a good immune activity [57]. The results of TIAN showed that Poria polysaccharide could exert immunomodulatory activity through TLR4/TRAF6/ NF- $\kappa \mathrm{B}$ signaling pathway [77]. Pu et al. found that Poria polysaccharide could exert immunomodulatory effects in $\mathrm{Ca}^{2+} / \mathrm{PKC} / \mathrm{p} 38 / \mathrm{NF}-\kappa \mathrm{B}$ signaling pathway [78].

All of the above studies confirm that Poria played an immunological regulatory role through various ways, which can lay a solid foundation for subsequent studies on immunity and benefit the exploitation of potential clinical application value.

3.3. Effects on Kidney. Studies have found that Poria can effectively resist renal injury, and the protection of diabetic nephropathy is a research hotspot. Li et al. study found that Poria polysaccharide treatment group could reduce serum IL-6 and TGR- $\beta 1$ in DN rats $(P<0.01)$, reduce inflammatory infiltration, and protect kidney tissue to a certain extent [79]. Wu et al. experiment showed that Poria polysaccharides could reduce hepatocyte apoptosis and inflammatory stress by inhibiting the NF- $\kappa$ B pathway, which indicated that Poria polysaccharides had a protective effect on acetaminophen-induced liver injury in mice [80]. Another experiment showed that WRP could enhance the antioxidant level by increasing superoxide dismutase and glutathione peroxidase and significantly reducing malondialdehyde level in mice kidney tissue. Another experiment showed that WRP could significantly reduce malondialdehyde level and enhance the antioxidant level of the body through increasing superoxide dismutase and glutathione peroxidase in kidney tissue of type 2 diabetic mice, and reduce the expression of the Bax gene in kidney tissue and reduce the apoptosis of renal tissue cells [58]. Zhang et al. experiment showed that WRP could inhibit the expression of the Bax gene in kidney tissue of type 2 diabetic mice and reduce the apoptosis of renal tissue cells. The mechanism of action still needs further study [81]. PPC could increase uric acid excretion by upregulating rOAT1 expression and downregulating rURAT1 expression. It was proved that PPC had anti-hyperuricemia activity [59].

Chen et al. study showed that Poricoic acid ZC (115), Poricoic acid ZD (116), and Poricoic acid ZE (69) could prevent tubulointerstitial fibrosis by blocking the interaction between TGFbR1 and Smad3, selectively inhibiting TGF $\beta 1$ and vaso-induced Smad3 phosphorylation [82]. Another of their experiments found that Poricoic acid ZG (117) and Poricoic acid ZH (20) could reduce renal fibrosis by inhibiting the TGF- $\beta /$ Smad pathway [83]. The above triterpenes were isolated for the first time and their activities were explored.

Studies showed that Poria had diuretic effect. The results of Yong et al. showed that poricoic acid A (94) had a significant diuretic effect on rats with water retention. The results showed that the urine volume of the poricoic acid A group was greater than the spironolactone group in the first hour, indicating that the effect of poricoic acid A was good for the diuretic effect. Reabsorption of electrolyte $\mathrm{Na}^{+}$and water increases urination [84]. Ni et al. selected triterpenoids extracted from Poria as ligands and selected three aquaporins AQP1, 4, 5 as target proteins. The results of screening with software and molecular docking showed that methyl dehydroabietate had a strong binding activity with AQP1, 4, and 5 , respectively. It was speculated that dehydroabietic acid methyl ester (137) could be the active substance of Poria for diuresis and spleen strengthening, which provided a reference for the follow-up study of active ingredients [85]. Intravenous injection of Poria aqueous extract $(1.5 \mathrm{~g} / \mathrm{kg})$ 
TABle 3: Summary table of Poria cocos activity.

\begin{tabular}{|c|c|c|}
\hline $\begin{array}{l}\text { Type of the } \\
\text { activities }\end{array}$ & Subjects & Activities \\
\hline & Total triterpenoids & $\begin{array}{c}\text { In vitro, the concentration of } 80 \mu \mathrm{g} / \mathrm{mL} \\
\text { extract could induce RKO cell line } \\
\text { apoptosis, } \mathrm{IC}_{50} \text { was } 34.14 \mu \mathrm{g} / \mathrm{mL}\end{array}$ \\
\hline & Triterpenes & $\begin{array}{l}\text { In vitro, the concentration of } 30 \mu \mathrm{g} / \mathrm{mL} \\
\text { extract could inhibit the proliferation of } \\
\text { A549 cell line, } \mathrm{IC}_{50} \text { was } 109.9 \mu \mathrm{g} / \mathrm{mL}\end{array}$ \\
\hline & $\mathrm{PA}$ & $\begin{array}{l}\text { In vitro, compared with } 0 \mu \mathrm{g} / \mathrm{mL} \text { group, } \\
10,20,40 \text {, and } 80 \mu \mathrm{g} / \mathrm{mL} \text { groups could } \\
\text { significantly increase the apoptosis rate } \\
\text { of } 786-0 \text { renal carcinoma cells }\end{array}$ \\
\hline
\end{tabular}

PA In vitro, $20.0 \mu \mathrm{mol} / \mathrm{L}$ PA promoted the

$\mathrm{PA}$ the proliferation and induced apoptosis

Poria ethanol extract

Antitumor action

PA

PA reduce the proliferation of

PAC and increase the apoptosis rate of

FMGP $\quad 400 \mu \mathrm{g} / \mathrm{mL}$ significantly inhibited the

PPSW-1 and Sul-W-1

Total triterpenes of Poria

Immune regulation

S-CMP

Poria polysaccharide

Poria polysaccharide apoptosis of Caski cells

In vitro, $1,2,5 \mu \mathrm{mol} / \mathrm{L}$ PAC inhibited of MDA-MB-231 cells

Poria ethanol extract (at $150 \mu \mathrm{g} / \mathrm{mL}$ )

could induce apoptosis of MDA-MB231 cells with $\mathrm{IC}_{50}$ value of $2.13 \pm 0.34 \mu \mathrm{g} / \mathrm{mL}$

In vitro, $\mathrm{PA}$ inhibited the proliferation of sgc-70901 cells at the concentrations of $0,20,40$, and $80 \mu \mathrm{M}$ osteosarcoma cells

In vitro, $\mathrm{PAC}(30,40,50 \mathrm{mg} / \mathrm{mL})$ can significantly reduce the migration rate human cancer HeLa cells ( $\mathrm{IC}_{50}$ is $60 \mathrm{mg} / \mathrm{mL}$ )

In vitro, the concentration of FMGP at migration of highly metastatic human lung cancer cell line CL1-5 cells

In vitro, the $\mathrm{IC}_{50}$ value was $26.34 \pm 0.77$, and the concentration of CMP3 was $100 \mu \mathrm{g} / \mathrm{mL}$, which had the highest inhibitory rate on HepG2 cells The concentration of PPSW-1 and Sul$\mathrm{W}-1$ at $100 \mu \mathrm{g} / \mathrm{mL}$ had a strong inhibitory effect on the migration of MDA-MB-231 cells in vitro

Total triterpenes of Poria can improve the immune function of mice in vitro

(at $40,20,10 \mu \mathrm{g} / \mathrm{mL}$ ) and in vivo (at $400,200,100 \mathrm{mg} / \mathrm{kg}$ )

S-CMP (at 100, $200 \mathrm{mg} / \mathrm{kg}$ ) showed immunoactivity in BALB/c mice Poria polysaccharide has

Inhibits the proliferation of colon cancer RKO cells and induces the apoptosis of colon cancer RKO cells through the mitochondrial apoptosis pathway

Inhibition of the Nrf2-ARE signaling pathway can prolong the duration of metastasis from early to advanced lung cancer

Inhibition of Wnt signaling pathway induced apoptosis in 786-0 renal carcinoma cells

Inhibit the survival of cervical cancer

Caski cells and promote apoptosis by inhibiting TRIM29 expression and downregulating Wnt pathway activity

The mechanism of action is related to the activation of PARP

By inducing mitochondria and death receptors to mediate apoptosis; the arrest of the G0/G1 cell cycle promotes apoptosis

Block the G0/G1 cell cycle

Apoptosis is mediated in part by the PTEN/Akt signaling pathway and caspase $3 / 7$ activity

The proapoptotic mechanism may be related to inhibition of phosphating of the ERK signaling pathway

By inhibiting the TGF $\beta$ RI mediated signaling pathway

Apoptosis is induced through the mitochondrial pathway and the death receptor pathway

Inhibition of the expression of the SATB1 gene reduces the migration ability of cancer cells immunomodulatory activity in vivo (at $200 \mathrm{mg} / \mathrm{kg}$ ) and in vitro (at $200 \mathrm{~g} / \mathrm{mL}$ ) Poria polysaccharide had $-$

Immunoregulatory activity is exerted through TLR4/TRAF6/NF- $\kappa$ B

signaling pathway in vitro and in vivo Immunoregulatory activity is exerted through $\mathrm{Ca}^{2+} / \mathrm{PKC} / \mathrm{p} 38 / \mathrm{NF}-\kappa \mathrm{B}$ signaling pathway in macrophages 
TABle 3: Continued.

\begin{tabular}{|c|c|c|c|c|}
\hline $\begin{array}{l}\text { Type of the } \\
\text { activities }\end{array}$ & Subjects & Activities & Mechanisms & Ref. \\
\hline \multirow{10}{*}{ Effects on kidney } & Poria polysaccharide & $\begin{array}{l}\text { Poria polysaccharide (at } 50,100, \\
200 \mathrm{mg} / \mathrm{kg} \text { ) significantly reduced the } \\
\text { inflammatory response of diabetic } \\
\text { nephropathy rats in vivo }\end{array}$ & - & {$[79]$} \\
\hline & Poria polysaccharides & $\begin{array}{c}\text { In vivo Poria polysaccharides (at } 200 \text {, } \\
400 \mathrm{mg} / \mathrm{kg} \text { ) had a protective effect on } \\
\text { acetaminophen-induced liver injury in } \\
\text { mice }\end{array}$ & $\begin{array}{c}\text { Inhibition of hepatocyte apoptosis } \\
\text { and inflammatory stress induced by } \\
\text { NF- } \kappa \text { B pathway plays a protective role } \\
\text { in the kidney }\end{array}$ & {$[80]$} \\
\hline & WRP & $\begin{array}{l}\text { In vivo, WRP }(200 \mathrm{mg} / \mathrm{kg}) \text { can inhibit } \\
\text { the trend of renal cell apoptosis in the } \\
\text { diabetic states }\end{array}$ & $\begin{array}{c}\text { Inhibition of Bax gene overexpression } \\
\text { in renal tissue decreased apoptosis of } \\
\text { renal cells }\end{array}$ & {$[58]$} \\
\hline & Pachymaran & $\begin{array}{l}\text { Pachymaran can prevent renal } \\
\text { interstitial fibrosis in rats with type } 2 \\
\text { diabetic nephropathy in vivo at doses of } \\
3,6 \text {, and } 12 \mathrm{mg} / \mathrm{kg} \text {, respectively }\end{array}$ & 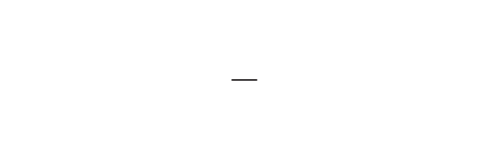 & {$[81]$} \\
\hline & PPC & $\begin{array}{c}\text { In vivo, } \mathrm{PPC} \text { at } 2 \mathrm{~g} / \mathrm{kg} \text { has anti- } \\
\text { hyperuricemia activity }\end{array}$ & $\begin{array}{l}\text { Uric acid excretion was increased by } \\
\text { upregulating rOAT1 expression and } \\
\text { downregulating rURAT1 expression }\end{array}$ & {$[59]$} \\
\hline & $\begin{array}{c}\text { Poricoic acid ZC, Poricoic } \\
\text { acid ZD, and Poricoic acid } \\
\text { ZE }\end{array}$ & $\begin{array}{c}\text { Poricoic acid ZC, poricoic acid ZD, and } \\
\text { Poricoic acid ZE can prevent } \\
\text { tubulointerstitial fibrosis in vivo (at } \\
10 \mathrm{mg} / \mathrm{kg} \text { ) and in vitro (at } 10 \mu \mathrm{M} \text { ) }\end{array}$ & $\begin{array}{l}\text { By inhibiting the activation of the } \\
\text { Wnt } / \beta \text {-catenin pathway and blocking } \\
\text { Smad } 3 \text { phosphorylation, renal } \\
\text { tubulointerstitial fibrosis was reduced }\end{array}$ & {$[82]$} \\
\hline & $\begin{array}{l}\text { Poricoic acid } \mathrm{ZG} \text { and } \\
\text { Poricoic acid } \mathrm{ZH}\end{array}$ & $\begin{array}{c}\text { Poricoic acid ZG and poricoic acid } \mathrm{ZH} \\
(\text { at } 10 \mu \mathrm{M}) \text { can inhibit renal fibrosis in } \\
\text { vitro }\end{array}$ & $\begin{array}{l}\text { Attenuate renal fibrosis via a Wnt/ } \\
\beta \text {-catenin pathway and targeted } \\
\text { phosphorylation of smad3 signaling }\end{array}$ & {$[83]$} \\
\hline & Poricoic acid A & $\begin{array}{c}\text { Poricoic acid } A \text { (at } 5,10,20 \mathrm{mg} / \mathrm{kg} \text { ) has } \\
\text { diuretic activity in vivo }\end{array}$ & - & {$[84]$} \\
\hline & $\begin{array}{l}\text { Dehydroabietic acid methyl } \\
\text { ester }\end{array}$ & $\begin{array}{l}\text { The authors found that methyl } \\
\text { dehydroabietic acid may be the diuretic } \\
\text { substance of Poria }\end{array}$ & - & {$[85]$} \\
\hline & Poria aqueous extract & $\begin{array}{l}\text { In vivo, Poria aqueous extract can } \\
\text { increase the urine volume of rabbits }\end{array}$ & - & {$[86]$} \\
\hline \multirow[t]{2}{*}{$\begin{array}{l}\text { Hepatoprotective } \\
\text { activity }\end{array}$} & Poria polysaccharides & $\begin{array}{l}\text { Effects of Poria polysaccharides on liver } \\
\text { protection against acetaminophen- } \\
\text { injured hepatocytes in vitro (at } 200 \text { and } \\
400 \mathrm{mg} / \mathrm{kg} \text { ) and in vivo (at } 20 \text { and } \\
40 \mathrm{~g} / \mathrm{L})\end{array}$ & $\begin{array}{c}\text { Through the molecular mechanisms } \\
\text { of reducing hepatocellular } \\
\text { inflammatory stress and Hsp90 } \\
\text { bioactivity }\end{array}$ & {$[87]$} \\
\hline & Carboxymethyl pachyman & $\begin{array}{l}\text { Carboxymethyl pachyman in vivo (at } \\
50 \mathrm{mg} / \mathrm{kg} \text { ) can alleviate liver injury of } \\
\text { CT26 mice induced by } 5 \text {-FU }\end{array}$ & $\begin{array}{l}\text { Hepatoprotective activity through } \\
\text { regulation of NF- } \kappa \mathrm{B}, \mathrm{Nrf} 2 \text {-ARE and } \\
\text { MAPK/P38/JNK pathways }\end{array}$ & {$[88]$} \\
\hline \multirow{3}{*}{$\begin{array}{l}\text { Effects on blood } \\
\text { sugar }\end{array}$} & Pachymic acid & $\begin{array}{c}\text { In vitro pachymic acid (at } 1 \mu \mathrm{M}) \text { can } \\
\text { increase glucose uptake in } 3 \mathrm{~T} 3-\mathrm{L} 1 \\
\text { adipocytes }\end{array}$ & $\begin{array}{l}\text { Hypoglycemic activity through } \\
\text { regulation of PI3K and AMPK } \\
\text { pathways }\end{array}$ & [89] \\
\hline & WRP & $\begin{array}{l}\text { WRP (at } 200 \mathrm{mg} / \mathrm{kg} \text { ) had hypoglycemic } \\
\text { effects on NIDDM mice in vitro }\end{array}$ & - & {$[90]$} \\
\hline & Insoluble polysaccharide & $\begin{array}{l}\text { Insoluble polysaccharide (at } 1.0 \mathrm{~g} / \mathrm{kg} \\
\text { and } 0.5 \mathrm{~g} / \mathrm{kg} \text { ) can improve the } \\
\text { symptoms of hyperglycemia in ob/ob } \\
\text { mice in vivo }\end{array}$ & $\begin{array}{l}\text { Hypoglycemic activity through } \\
\text { regulation of intestinal flora }\end{array}$ & [91] \\
\hline
\end{tabular}


TABle 3: Continued.

\begin{tabular}{|c|c|c|c|c|}
\hline $\begin{array}{l}\text { Type of the } \\
\text { activities }\end{array}$ & Subjects & Activities & Mechanisms & Ref. \\
\hline \multirow[t]{3}{*}{ Antioxidant effects } & $\begin{array}{l}\text { Carboxymethyl sulfate Poria } \\
\text { polysaccharide }\end{array}$ & $\begin{array}{c}\text { Carboxymethyl sulfate Poria } \\
\text { polysaccharide had the strongest } \\
\text { scavenging effect on } \mathrm{OH} \text { and } \mathrm{O}_{2}^{-} \text {and } \\
\text { there was an agent-activity relationship. } \\
\text { When the sample concentration was } \\
4.5 \mathrm{mg} / \mathrm{mL} \text {, the scavenging rates of } \mathrm{OH} \\
\text { and } \mathrm{O}_{2}^{-} \text {were } 79 \% \text { and } 84.2 \% \text {, } \\
\text { respectively, which indicated that the } \\
\text { sample had a certain antioxidant } \\
\text { activity. }\end{array}$ & - & {$[64]$} \\
\hline & PCP-M & $\begin{array}{l}\text { PCP-M polysaccharides (at } 2.0 \mathrm{mg} / \mathrm{mL} \text { ) } \\
\text { had antioxidant activity in vitro }\end{array}$ & - & {$[60]$} \\
\hline & Carboxymethyl-pachyman & $\begin{array}{l}\text { In vivo, carboxymethyl-pachyman (at } \\
200 \mathrm{mg} / \mathrm{kg} \text { ) has antioxidant activity }\end{array}$ & - & [92] \\
\hline
\end{tabular}
showed anti-inflammatory activity in vitro

In vitro $\mathrm{PA}$ (at $25,50,100 \mathrm{mg} / \mathrm{L}$ )

PA

Anti-inflammatory effects

Poricoic acid C

CMP33

Poria polysaccharide

$16 \alpha$-Hydroxytrametenolic Acid

Poria ethanol extract

Effects on the gut

Poria powder and watersoluble polysaccharide

Poria powder

Sulfated pachymaran

Antidepressant

inhibits TNF- $\alpha$-induced inflammation and oxidative stress damage in $\mathrm{SH}-$ SY5Y

Poricoic acid C $(50,100 \mu \mathrm{M})$ had antiinflammatory activity on RAW264.7 cells stimulated by LPS in vitro

CMP33 $(62.5-1000 \mu \mathrm{g} / \mathrm{mL})$ has antiinflammatory activity in vitro

In vivo, Poria polysaccharide (at 5, 100, $200 \mathrm{mg} / \mathrm{kg}$ ) can reduce the infiltration degree of colitis

In vitro, $16 \alpha$-hydroxytrametenolic Acid $(60 \mu \mathrm{M})$ can improve intestinal barrier function

Poria ethanol extract (at $32 \mathrm{~g} / \mathrm{mL}$ ) inhibited intestinal contraction in vitro

In vivo, Poria powder (at $2.0 \mathrm{~g} / \mathrm{kg}$ ) and water-soluble polysaccharide (at

$7.6 \mathrm{mg} / \mathrm{kg}$ ) can protect against intestinal damage caused by cisplatin Poria powder (at $50 \mu \mathrm{g} / \mathrm{kg}$ ) can increase the level of intestinal bifidobacteria in mice in vivo

In vivo, sulfated pachymaran $(25 \mathrm{mg} / \mathrm{kg}$,

$50 \mathrm{mg} / \mathrm{kg}, 100 \mathrm{mg} / \mathrm{kg}$ ) had an antidepressant effect compared with the depression model group

In vivo, PCWPW and PCWPS $(300 \mathrm{mg} / \mathrm{kg})$ possess antidepressantlike effects
Anti-inflammatory effects through downregulation of iNOS and COX-2 expression and inhibition of $\mathrm{NO}$ and PGE2 production

It may be a mechanism of action to inhibit apoptosis by downregulating

Nrf2 of the ERK/Nrf2 signaling pathway into the nucleus

Inhibition of iNOS and COX-2

expression through downregulation of NF- $\kappa \mathrm{B}$ exerts anti-inflammatory effects

Anti-inflammatory activity by inhibiting the overproduction of $\mathrm{NO}$,

IL-6, TNF- $\alpha$, and IL- $1 \beta$ in LPSstimulated RAW264.7 cells

Anti-inflammatory activity through inhibition of IL-33/ST2 signaling pathway activation

Improving intestinal barrier function via $\mathrm{PI} 3 \mathrm{~K} / \mathrm{Akt} / \mathrm{NF}-\kappa \mathrm{B}$ pathway

Inhibits spontaneous and spastic contractions of the small intestine by inhibiting $M$ receptors and regulating potassium and calcium channels Water-soluble polysaccharides exert enteroprotective activity through intestinal flora and metabolic regulation

Antidepressant activity through increased protein expression of $\mathrm{p}$ CREB and BDNF

PCWPW and PCWPS 
TABLE 3: Continued.

\begin{tabular}{|c|c|c|c|c|}
\hline $\begin{array}{l}\text { Type of the } \\
\text { activities }\end{array}$ & Subjects & Activities & Mechanisms & Ref. \\
\hline \multirow{7}{*}{$\begin{array}{l}\text { Other biological } \\
\text { activities }\end{array}$} & $\begin{array}{l}\text { Total triterpenes and } \\
\text { poricoic acid A }\end{array}$ & $\begin{array}{l}\text { In vitro, the effects of total triterpenes } \\
\text { and poricoic acid A on the inhibition of } \\
\text { tyrosinase activity in cells increased } \\
\text { with a rising concentration of } \\
\qquad 12.5 \mu \mathrm{g} / \mathrm{mL}\end{array}$ & $\begin{array}{c}\text { Pharmacological activity through } \\
\text { inhibition of monophenolic and } \\
\text { diphenolic enzyme activities in } \\
\text { tyrosinase }\end{array}$ & [99] \\
\hline & Poria ethanol extract & $\begin{array}{l}\text { Poria ethanol extract (at } 100 \mu \mathrm{g} / \mathrm{mL} \text { ) } \\
\text { can reduce the expression of tyrosinase } \\
\text { and MITF in B16F10 in vitro, and } \\
\text { applying } 2 \% \text { ointment containing Poria } \\
\text { extract on cheeks in vivo can reduce the } \\
\text { skin color value }\end{array}$ & $\begin{array}{l}\text { By regulating tyrosinase activity and } \\
\text { MITF expression to reduce the } \\
\text { production of melanin }\end{array}$ & {$[100]$} \\
\hline & & & $\begin{array}{l}\text { Poria chloroform extract can inhibit } \\
\text { the activity of tyrosinase in the }\end{array}$ & \\
\hline & Poria chloroform extract & $\begin{array}{l}\text { Poria chloroform extract (at } 2 \sim 3 \mathrm{mg} / \\
\mathrm{mL} \text { ) has a strong tyrosinase inhibition } \\
\text { effect in vitro }\end{array}$ & $\begin{array}{l}\text { process of melanin production and } \\
\text { effectively regulate the production of } \\
\text { melanin, which is suitable for the } \\
\text { development of whitening products } \\
\text { for treating pigmented skin diseases }\end{array}$ & {$[101]$} \\
\hline & PA & $\begin{array}{c}\text { PA }(100 \mathrm{mg} / \mathrm{kg}) \text { has significant } \\
\text { neuroprotective effects on cerebral } \\
\text { ischemia/reperfusion injury and } \\
\text { neuronal apoptosis in vivo }\end{array}$ & $\begin{array}{l}\text { The mechanism of action may be } \\
\text { related to the activation of the PI } 3 \mathrm{~K} / \\
\text { Akt signaling pathway }\end{array}$ & {$[102]$} \\
\hline & $\begin{array}{l}\text { Total triterpenes in Poria } \\
\text { cocos peel (TTP) }\end{array}$ & $\begin{array}{c}\text { In vivo, TTP }(100 \mathrm{mg} / \mathrm{kg}) \text { has } \\
\text { antiepileptic activity }\end{array}$ & - & {$[103]$} \\
\hline & $\begin{array}{l}\text { Poria aqueous extract, } \\
\text { alcohol extract, and Poria } \\
\text { polysaccharide }\end{array}$ & $\begin{array}{l}\text { In vivo, Poria aqueous extract (at } 30,60 \text {, } \\
120 \mathrm{mg} / \mathrm{kg} \text { ), Poria alcoholic extract (at } \\
25,50,100 \mathrm{mg} / \mathrm{kg} \text { ), and Poria } \\
\text { polysaccharide (at } 10,20,40 \mathrm{mg} / \mathrm{kg} \text { ) } \\
\text { protect against acute liver injury caused } \\
\text { by carbon tetrachloride }\end{array}$ & $\begin{array}{l}\text { Protects the liver by enhancing the } \\
\text { liver's antioxidant capacity and } \\
\text { reducing inflammation }\end{array}$ & {$[104]$} \\
\hline
\end{tabular}

increased the urine volume in rabbits within 20 and 30 minutes, which was much higher than that of the control groups [86].

3.4. Hepatoprotective Activity. Wu et al. research demonstrated that Poria polysaccharides could reduce the inflammatory stress of liver cells and the biological activity of HSP90, which proved that Poria polysaccharides had a liver protective effect against acetaminophen-damaged liver cells [87]. Wang et al. found that carboxymethyl pachyman could reduce liver injury of CT2 6 mice by regulating NF- $\kappa \mathrm{B}, \mathrm{Nrf2}-$ ARE, and MAPK/P38/JNK pathways [88].

3.5. Effects on Blood Sugar. Sun et al. proved that pachymic acid could stimulate glucose uptake in 3T3-L1 adipocytes by enhancing GLUT4 expression and transport [89]. Not only that, PAC could also reduce blood glucose in diabetic rats [90]. Sun et al. reported for the first time that insoluble polysaccharide could improve and regulate hyperglycemia and hyperlipidemia in ob/ob mice through intestinal flora [91].

3.6. Antioxidant Effects. Wang et al. experiment showed that PCP-M had the scavenging ability of hydroxyl radical and
DPPH radical [60]. Zhang et al. experiment showed that carboxymethyl-pachyman could reduce the generation of MDA in liver tissue and serum of mice and increase the activity of SOD in serum and liver, which indicated that carboxymethyl-pachyman had antioxidant activity [92].

3.7. Anti-Inflammatory Effects. A large number of studies have shown that Poria has anti-inflammatory activity. Five compounds were isolated from Poria by Rak et al. They were poricoic acid A (94), 3-O-acetyl-16 $\alpha$-hydroxydehydrotrametenolic acid (47), polyporenic acid C (53), 3 $\beta$ hydroxylanosta-7,9(11),24-trien-21-oic acid(39), and trametenolic acid (3). These compounds could downregulate the expression of COX-2 and PGE2 by inhibiting the production of NO and the expression of iNOS in RAW264.7 cells stimulated by LPS; poricoic acid A exerted the highest anti-inhibitory activity and reduced PGE2 levels via downregulation of COX-2 protein expression, indicating that they had anti-inflammatory activities [93]. Qin's study found that PA could inhibit TNF- $\alpha$ induced oxidative stress and inhibit apoptosis of SH-SY5Y cells by inhibiting ERK/ Nrf2 pathway [94].

Coriacoic acid A (131), Coriacoic acid B (73), dehydroeburic acid (46), acetyl eburicoic acid (19), and Poricoic acid C (97) could inhibit NO production, among which the 
activities of Poricoic acid $\mathrm{C}$ were the strongest. Its mechanism was to exert anti-inflammatory activity by downregulating NF-kappaB to inhibit the expression of iNOS and COX-2. Coriacoic acid A and Coriacoic acid B were isolated for the first time and found to have anti-inflammatory activity for the first time [30]. CMP33 (35) could inhibit the release of NO, IL-1 $\beta$, IL-6, and TNF- $\alpha$ in RAW264.7 macrophages stimulated by LPS, indicating that PPS had anti-inflammatory activity [51]. Liang et al. found that Poria polysaccharide could inhibit the activation of IL-33/ST2 signaling pathway, reduce the activation of UC, inhibit the expression of inflammatory factors, and reduce the infiltration degree of colitis, which indicated that Poria polysaccharide had an obvious therapeutic effect on ulcerative colitis [95].

3.8. Effects on the Gut. Studies showed that Poria had a protective effect on the intestinal tract. 16 $\alpha$-hydroxytrametenolic acid (6) could improve intestinal barrier function through glucocorticoid receptor-mediated PI3K/ Akt/NF- $\kappa \mathrm{B}$ pathway, suggesting that $16 \alpha$-hydroxytrametenolic acid could strengthen the intestinal barrier [96]. Xiao et al. study showed that the alcohol extract of Poria could inhibit intestinal contraction in vitro by blocking the $\mathrm{M}$ receptor and regulate intestinal peristalsis function, which provided a new theoretical basis for the treatment of diarrhea type IBS [97]. Zou showed that watersoluble polysaccharides could increase the relative content of probiotic bacteria and decrease the relative content of pathogenic bacteria to regulate the change of intestinal flora structure caused by cis-uranium, and water-soluble polysaccharides could also reduce the intestinal damage caused by cis-uranium by regulating the disturbance of metabolic pathways such as lipid metabolism, amino acid metabolism, and purine metabolism [16]. The experimental results of Song et al. showed that Poria powder exerted a regulatory effect on intestinal flora by significantly increasing the level of intestinal bifidobacteria in mice [98].

3.9. Antidepressant. Poria has antidepressant activity. Zhang et al. study demonstrated that sulfated pachymaran had antidepressant-like effects in rats, which may be mediated by enhancing GluR1 receptor function and upregulating the protein expression of $\mathrm{p}-\mathrm{CREB}$ and BDNF in the hippocampus [61]. Zhang et al. experiments showed that the resting time of animals treated with $300 \mathrm{mg} / \mathrm{kg}$ PCWPW and PCWPS was also significantly shortened $(P<0.001)$, suggesting that PCWPW and PCWPS have antidepressant effects. PCWPs had a good protective effect on $\mathrm{H}_{2} \mathrm{O}_{2}$-induced cell death in vitro. Its neuroprotective effect could reduce nerve damage in patients with depression [62].

3.10. Other Biological Activities. Poria also showed effect on tyrosinase activity [99-101]. In addition, pachymic acid (1) had protective effects against cerebral ischemia-reperfusion injury and neuronal apoptosis [102]; epidermis extract could be a potential treatment for epilepsy [103]. Poria's aqueous extract, alcohol extract, and polysaccharide showed the protective effects on acute liver injury caused by carbon tetrachloride [104].

\section{Conclusion and Prospect}

In recent years, many researches have been conducted on the extracts of Poria and their multiple biological activities. Poricoic acid A (95), for example, not only showed its impact on the tyrosinase activity but also has a diuretic effect. These active compounds have enormous potential to be developed to treat some diseases with multi-targets safely and effectively. In this paper, both the chemical composition and biological activity of Poria were discussed in detail to provide abundant theoretical guidance for the further development of Poria as a potential medicinal and edible resource.

\section{Conflicts of Interest}

The authors declare no conflicts of interest.

\section{Acknowledgments}

This work was supported by National Key R\&D Program of China (2018YFD0400200), Major Public Welfare Projects in Henan Province (201300110200), and the Key Project in Science and Technology Agency of Henan Province (192102110214 and 202102110283).

\section{References}

[1] P. F. Yang, P. C. Zhang, and R. Y. Chen, "Research advances on chemical constituents and bioactivities of Poria cocos (Schw.) Wolf," in Proceedings of the First National Poria cocos Conference 2014, Wu Han, China, October 2014.

[2] M. Wang, Effective Constituents and Mechanism of the Surface Layer of Poria cocos against Renal Fibrosis, Northwest University, Xian, China, 2019.

[3] G. P. Yang, "Poria cocos development prospect is broad," Hunan Linye, vol. 38, no. 2, p. 38, 2018.

[4] P. P. Sun, Study on the Pharmaceutical Research of Fuling San, Hubei University of Chinese Medicine, Wuhan, China, 2013.

[5] Y. Y. Shao, D. Zhu, G. H. Yang et al., "Recent advances in research on edible Poria cocos," in Proceedings of the 9th Annual Conference of Chinese Medicine Chemistry Branch, Chinese Society of Traditional Chinese Medicine, Xiamen, China, July 2014.

[6] L. Ma, L. Yin, B. Wang et al., "Reasarch progress of Poria cocos," Asian-Pacific Traditional Medicine, vol. 11, no. 12, pp. 55-59, 2015.

[7] Z. P. Liang, "Advance in research on chemical constituents, pharmacological effects and clinical applications of Poria cocos," China's Urban and Rural Enterprise Health, vol. 33, no. 8, pp. 51-53, 2018.

[8] X. L. Zhuang, "Value of clinical application of traditional Chinese medical Poria cocos," Smart Healtheare, vol. 4, no. 5, pp. 86-87, 2018.

[9] T. Tai, A. Akahori, and T. Shingu, "Triterpenes of Poria cocos," Phytochemistry, vol. 32, no. 5, pp. 1239-1244, 1993.

[10] Z. J. Zhōng, "Ingredients of Poria cocos (first report)," Yakugaku Zasshi, vol. 59, no. 10, pp. 725-729, 1939. 
[11] A. Ken, "China root triterpenoid (1st report)," Yakugaku Zasshi, vol. 90, no. 4, pp. 475-479, 1970.

[12] L. Y. Wang and H. J. Wan, "Studies on the chemical constituents of fuling (Poria cocos)," Chinese Traditional and Herbal Drugs, vol. 29, no. 3, pp. 145-148, 1998.

[13] H. Z. Liu, S. L. Wu, D. Y. Zhang et al., "A review on separation and medicinal value of active pharmaceutical ingredients from Poria cocos," Edible Fungi of China, vol. 34, no. 6, pp. 1-6, 2015.

[14] A. Kanematsu and S. Natori, "Triterpenoids of Hoelen (fuling), sclerotia of Poria cocos (Schw.) Wolf. I," Yakugaku Zasshi, vol. 90, no. 4, pp. 475-479, 1970.

[15] T. Tai, T. Shingu, T. Kikuchi et al., "Triterpenes from the surface layer of Poria cocos," Phytochemistry, vol. 39, no. 5, pp. 1165-1169, 1995.

[16] Y. T. Zou, Characterization of Chemical Constituents of Poria cocos and Primary Study of their Protective Effects against Cisplatin-Induced Intestinal Injury, Nanjing University of Chinese Medicine, Nanjing, China, 2019.

[17] T. Akihisa, E. Uchiyama, T. Kikuchi, H. Tokuda, T. Suzuki, and Y. Kimura, "Anti-tumor-promoting effects of 25methoxyporicoic acid A and other triterpene acids from Poria cocos," Journal of Natural Products, vol. 72, no. 10, pp. 1786-1792, 2009.

[18] K.-H. Lai, M.-C. Lu, Y.-C. Du et al., "Cytotoxic lanostanoids from Poria cocos," Journal of Natural Products, vol. 79, no. 11, pp. 2805-2813, 2016.

[19] D. Lee, S. Lee, S. H. Shim et al., "Protective effect of lanostane triterpenoids from the sclerotia of Poria cocos Wolf against cisplatin-induced apoptosis in LLC-PK1 cells," Bioorganic \& Medicinal Chemistry Letters, vol. 27, no. 13, pp. 2881-2885, 2017.

[20] S. Wang, Y. Y. Jiang, N. L. Zhu et al., "Determination and isolation of the chemical constituents of Poria cocos," Journal of Beijing University of Traditional Chinese Medicine, vol. 33, no. 12, pp. 841-844, 2010.

[21] T. Chen, L. Hua, G. Chou, X. Mao, and X. Zou, “A unique naphthone derivative and a rare 4,5-seco-lanostane triterpenoid from Poria cocos," Molecules, vol. 23, no. 10, p. 2508, 2018.

[22] T. Shingu, T. Tai, and A. Akahori, "A lanostane triterpenoid from Poria cocos," Phytochemistry, vol. 31, no. 7, pp. 2548-2549, 1992.

[23] T. Akihisa, Y. Mizushina, M. Ukiya et al., "Dehydrotrametenonic acid and dehydroeburiconic acid from Poria cocos and their inhibitory effects on eukaryotic DNA polymerase $\alpha$ and $\beta$," Bioscience, Biotechnology, and Biochemistry, vol. 68, no. 2, pp. 448-450, 2004.

[24] N. Zhang, Z. X. Li, J. Li et al., "Advances in the research of constituents and pharmacological effects of Poria cocos (Schw.) Wolf," Modernization of Traditional Chinese Medicine and Materia Medica-World Science and Technology, vol. 21, no. 2, pp. 220-233, 2019.

[25] T. Akihisa, Y. Nakamura, H. Tokuda et al., "Triterpene acids from Poria cocos and their anti-tumor-promoting effects," Journal of Natural Products, vol. 70, no. 6, pp. 948-953, 2007.

[26] Y. Zheng and X. W. Yang, "Poriacosones A and B: two new lanostane triterpenoids from Poria cocos," Journal of Asian Natural Products Research, vol. 10, no. 7-8, pp. 645-651, 2008.

[27] T. Tai, A. Akahori, and T. Shingu, "Triterpenoids from Poria cocos," Phytochemistry, vol. 30, no. 8, pp. 2796-2797, 1991.

[28] T.-G. Cai and Y. Cai, "Triterpenes from the fungus Poria cocos and their inhibitory activity on nitric oxide production in mouse macrophages via blockade of activating protein-1 pathway," Chemistry \& Biodiversity, vol. 8, no. 11, pp. 2135-2143, 2011.

[29] H. J. Dong, Antitumor Activities of Triterpenes Isolated from the Epidermis of Poria cocos (Schw). Wolf, China Academy of Chinese Medical Sciences, Beijing, China, 2015.

[30] S. Lee, D. Lee, S. O. Lee et al., "Anti-inflammatory activity of the sclerotia of edible fungus, Poria cocos Wolf and their active lanostane triterpenoids," Journal of Functional Foods, vol. 32, pp. 27-36, 2017.

[31] M. Wang, D. Q. Chen, M. C. Wang et al., "Poricoic acid ZA, a novel RAS inhibitor, attenuates tubulo-interstitial fibrosis and podocyte injury by inhibiting TGF- $\beta /$ Smad signaling pathway," Phytomedicine, vol. 36, pp. 243-253, 2017.

[32] C.-H. Yang, S.-F. Zhang, W.-Y. Liu, Z.-J. Zhang, and J.-H. Liu, "Two new triterpenes from the surface layer of Poria cocos," Helvetica Chimica Acta, vol. 92, no. 4, pp. 660-667, 2009.

[33] L. Yang, B. Qin, S. Feng, S. Liu, and X. Song, "A new triterpenoid from traditional Chinese medicine Poria cocos," Journal of Chemical Research, vol. 34, no. 10, pp. 553-554, 2010.

[34] S. Li, Z. Wang, R. Gu et al., "A new epidioxy-tetracyclic triterpenoid from Poria cocos Wolf," Natural Product Research, vol. 30, no. 15, pp. 1712-1717, 2016.

[35] H. Q. Lin, Y. Y. Song, and Y. C. Huang, "A 4,5-secolanostane triterpenoid from the sclerotium of Poria cocos," Journal of Medical Sciences, vol. 30, no. 6, p. 273, 2010.

[36] D. P. Li, X. Y. Liang, H. S. Chen et al., "Studies on the chemical composition of Poria cocos peel in yunnan," Guihaia, no. 3, pp. 3-5, 1998.

[37] L. Li, E. D. Yuan, N. Gou et al., "Effects of water extract of poria on Helicobacter pylori inhibition and GES-1 cells proliferation," Modern Food Science \& Technology, vol. 35, no. 10, pp. 19-24+147, 2019.

[38] B. Hu, Y. P. Yang, and Y. Ye, "Chemical constituents from Poria cocos (Schw.) Wolf," Chinese Traditional and Herbal Drugs, vol. 37, no. 5, pp. 655-658, 2006.

[39] G. C. Hoffmann, B. W. Simson, T. E. Timell et al., "Structure and molecular size of pachyman," Carbohydrate Research, vol. 20, no. 1, pp. 185-188, 1971.

[40] H. Kanayama, N. Adachi, and M. Togami, "A new antitumor polysaccharide from the mycelia of Poria cocos Wolf," Chemical and Pharmaceutical Bulletin, vol. 31, no. 3, pp. 1115-1118, 1983.

[41] L. N. Zhang, Q. Ding, P. Y. Zhang, and H.-Q. Feng, "Isolation and structural analysis of polysaccharides from the sclerotium of Poria cocos Wolf," Gaodeng Xuexiao Huaxue Xuebao, vol. 18, no. 6, pp. 990-993, 1997.

[42] Q. Ding, L. N. Zhang, and Z. Q. Zhang, "Isolation and structural analysis of polysaccharides from poria mycelia," Acta Polymerica Sinica, vol. 70, no. 2, pp. 224-227, 2000.

[43] L. N. Zhang, L. Chen, Y. Jin et al., "Comparision of polysaccharides isolated from the mycelia of a cultivated strain of Poria cocos grown in different LIQUID culture media," Acta Polymerica Sinica, vol. 21, no. 4, pp. 465-472, 2003.

[44] Y. Jin, L. Zhang, L. Chen, Y. Chen, P. C. Keung Cheung, and L. Chen, "Effect of culture media on the chemical and physical characteristics of polysaccharides isolated from Poria cocos mycelia," Carbohydrate Research, vol. 338, no. 14, pp. 1507-1515, 2003.

[45] Y. L. Lin, L. N. Zhang, Y. Jin et al., "Isolation, chemical components and molecular weights of polysaccharides from Poria cocos mycelia with cultivated strain," Acta Polymerica Sinica, no. 1, pp. 97-103, 2003. 
[46] Y. Wang, M. Zhang, D. Ruan et al., "Chemical components and molecular mass of six polysaccharides isolated from the sclerotium of Poria cocos," Carbohydrate Research, vol. 339, no. 2, pp. 327-334, 2004.

[47] Y. F. Wang and L. N. Zhang, "Chain conformation of carboxymethylated derivatives of (1 to 3 )- $\beta$-D-glucan from Poria cocos sclerotium," Carbohydrate Polymers, vol. 65, no. 4, pp. 504-509, 2007.

[48] Q. Huang, Y. Jin, L. Zhang, P. C. K. Cheung, and J. F. Kennedy, "Structure, molecular size and antitumor activities of polysaccharides from Poria cocos mycelia produced in fermenter," Carbohydrate Polymers, vol. 70, no. 3, pp. 324-333, 2007.

[49] X. Chen, Q. Tang, Y. Chen, W. Wang, and S. Li, "Simultaneous extraction of polysaccharides from Poria cocos by ultrasonic technique and its inhibitory activities against oxidative injury in rats with cervical cancer," Carbohydrate Polymers, vol. 79, no. 2, pp. 409-413, 2010.

[50] C. Huang, Y. M. Wang, and J. Zhao, "Preparation, purification, and analysis of chemical composition of anti-tumuor polysaccharide from Poria cocos," Chinese Traditional and Herbal Drgus, vol. 43, no. 11, pp. 2146-2149, 2012.

[51] X. F. Liu, X. Q. Wang, X. F. Xu, and X. Zhang, "Purification, antitumor and anti-inflammation activities of an alkalisoluble and carboxymethyl polysaccharide CMP33 from Poria cocos," International Journal of Biological Macromolecules, vol. 127, pp. 39-47, 2019.

[52] E. H. Tang and W. Cai, "Effects of pachymaran on proliferation, migration and pro-apoptosis of human cervical carcinoma HeLa cells and its mechanism," Cancer Research on Prevention and Treatment, vol. 46, no. 8, pp. 707-713, 2019.

[53] T.-Y. Lin, M.-K. Lu, and C.-C. Chang, "Structural identification of a fucose-containing 1,3- $\beta$-mannoglucan from Poria cocos and its anti-lung cancer CL1-5 cells migration via inhibition of TGF $\beta \mathrm{R}$-mediated signaling," International Journal of Biological Macromolecules, vol. 157, pp. 311-318, 2020.

[54] H. Z. Yang, F. Q. Xiong, L. Yang et al., "Effect of different components carboxymethyl pachyman (CMP) on HepG2 cell proliferation," Food and Nutrition in China, vol. 25, no. 12, pp. 26-28, 2019.

[55] B. Song, X. L. Li, Y. Z. Wu et al., "Study on preparation and anti-tumor activity of carboxymethyl pachymaran," Chinese Journal of Modern Applied Pharmacy, vol. 36, no. 11, pp. 1328-1332, 2019.

[56] K. Hu, Q. Luo, X. F. Zhu et al., "Isolation of homogeneous polysaccharide from Poria cocos and effect of its sulfated derivatives on migration of human breast cancer MDA-MB231 cells," China Journal of Chinese Materia Medica, vol. 44, no. 13, pp. 2835-2840, 2019.

[57] H. Wang, J. F. Mukerabigwi, Y. Zhang et al., "In vivo immunological activity of carboxymethylated-sulfated $(1 \rightarrow 3)$ $\beta$-d-glucan from sclerotium of Poria cocos," International Journal of Biological Macromolecules, vol. 79, pp. 511-517, 2015.

[58] C. L. Huang, J. L. Zheng, F. L. Li et al., "Effect of pachymaran to the antioxidant capacity and Bax, bcl-2 protein expression of renal tissue in mice with type H diabetes," Journal of Food Science and Biotechnology, vol. 35, no. 1, pp. 82-88, 2016.

[59] L. J. Deng, J. X. Yan, P. Wang et al., "Effects of pachman on the expression of renal tubular transporters rURAT1, rOAT1 and rOCT2 of the rats with hyperuricemia," Western Journal of Traditional Chinese Medicine, vol. 32, no. 6, pp. 10-14, 2019.
[60] N. Wang, Y. Zhang, X. Wang et al., “Antioxidant property of water-soluble polysaccharides from Poria cocos Wolf using different extraction methods," International Journal of Biological Macromolecules, vol. 83, pp. 103-110, 2016.

[61] J. Y. Zhang, J. Tang, Q. Zhang et al., "Effects of sulfated pachymaran AMPA receptor expression in hippocampi of depression model rats," Chinese Journal of Clinical Psychology, vol. 27, no. 6, pp. 1086-1091, 2019.

[62] W. Zhang, L. Chen, P. Li, J. Zhao, and J. Duan, "Antidepressant and immunosuppressive activities of two polysaccharides from Poria cocos (Schw.) Wolf," International Journal of Biological Macromolecules, vol. 120, pp. 16961704, 2018.

[63] L. Chen, Study on Optimization of Extraction Technology and Exploitation of Pachyman, Guizhou University, Guiyang, China, 2007.

[64] Y. Cao, Chemical Modification of Pachymaran and its Antioxidant Activity, Tianjin University of Science and Technology, Tianjin, China, 2010.

[65] H. G. Qiang and Z. Q. Yang, "Study on the anti-HIV effect of carboxymethyl pachymaran in vitro," China Medical Herald, no. 10, pp. 1156-1158, 2008.

[66] N. Wang, "Effect of sodium carboxymethyl pachymaran on type I human herpesvirus 1 in vitro," Journal of Aerospace Medicine, vol. 30, no. 8, pp. 933-934, 2010.

[67] Y. R. Feng, W. Liu, and J. G. Yang, "Preparation of carboxymethylated pachyman with different molecular weight and study on its antioxidative activity," China Food Additives, vol. 30, no. 3, pp. 67-74, 2019.

[68] J. Lin, Study of Immunosuppressive Activity and ApoptosisInducing Effect of Total Triterpenoids from Poria cocos on Human Colorestal Carcinoma RKO Cells, Southern Medical University, Guangzhou, China, 2016.

[69] P. Jin and F. P. Wu, "Regulation mechanism of triterpenes from Poria cocos in Jingzhou on Nrf2 signaling pathway in A549 cells," China Pharmaceuticals, vol. 28, no. 8, pp. 1-3, 2019.

[70] J. S. Dong, J. F. Zhao, L. C. Zhang et al., "Effect of pachymic acid on biological characteristics of renal carcinoma cells through Wnt signaling pathway," Chinese Journal of Gerontology, vol. 39, no. 9, pp. 2241-2244, 2019.

[71] L. Shen and D. H. Wong, "Pachymic acid regulated and controlled survival and apoptosis in cervical cancer cells through Wnt signaling pathway by inhibiting TRIM29 expression," Journal of Guangzhou University of Traditional Chinese Medicine, vol. 37, no. 1, pp. 140-146, 2020.

[72] W. Jiang, "Induction of pachymic acid on apoptosis of human breast cancer MDA-MB-231cells by activating poly ADP ribose polymerase," Chinese Traditional and Herbal Drgus, vol. 47, no. 21, pp. 3861-3865, 2016.

[73] Y. Jiang and L. Fan, "Evaluation of anticancer activities of Poria cocos ethanol extract in breast cancer: in vivo and in vitro, identification and mechanism," Journal of Ethnopharmacology, vol. 257, p. 112851, 2020.

[74] K. X. Sun and H. W. Xia, "Pachymic acid inhibits growth and induces cell cycle arrest and apoptosis in gastric cancer SGC7901 cells," Oncology Letters, vol. 16, no. 2, pp. 2517-2524, 2018.

[75] H. Wen, Z. Wu, H. Hu et al., "The anti-tumor effect of pachymic acid on osteosarcoma cells by inducing PTEN and Caspase 3/7-dependent apoptosis," Journal of Natural Medicines, vol. 72, no. 1, pp. 57-63, 2018.

[76] J. H. Xie, J. Lin, L. S. Lie et al., "Experimental study of the inhibitory effect of total triterpcnoids from Poria cocos on 
mouse immune response and therapeutic effect on rat adjuvant arthritis," Pharmacology and Clinics of Chinese Materia Medica, vol. 32, no. 6, pp. 89-92, 2016.

[77] H. Tian, Poria cocos Polysaccharide Plays an Immunomodulatory Role through TLR4/TRAF6/NF-B Signaling Pathway, Medical University, Chongqing, China, 2019.

[78] Y. Pu, Z. Liu, H. Tian, and Y. Bao, "The immunomodulatory effect of Poria cocos polysaccharides is mediated by the $\mathrm{Ca} 2+1$ $\mathrm{PKC} / \mathrm{p} 38 / \mathrm{NF}-\kappa \mathrm{B}$ signaling pathway in macrophages," International Immunopharmacology, vol. 72, pp. 252-257, 2019.

[79] J. W. Li, Y. Q. Pang, M. Zhou et al., "Effect of Poria cocos polysaccharide on inflammatory reaction in diabetic nephropathy rats," Diet Health, vol. 6, no. 28, pp. 64-65, 2019.

[80] K. Wu, J. L. Fan, X. Y. Huang et al., "Hepatoprotective effects exerted by Poria cocos polysaccharides against acetaminophen-induced liver injury in mice," International Journal of Biological Macromolecules, vol. 114, pp. 38-142, 2018.

[81] H. R. Zhang, X. H. Lv, X. Kang et al., "Effect of Poria cocos polysaccharide on renal interstitial fibrosis in rats with type 2 diabetic nephropathy," Contemporary Medicine, vol. 22, no. 8, pp. 1-2, 2016.

[82] D. Q. Chen, L. Chen, M. Wang et al., "Novel inhibitors of the cellular renin-angiotensin system components, poricoic acids, target Smad3 phosphorylation and $\mathrm{Wnt} / \beta$-catenin pathway against renal fibrosis," British Journal of Pharmacology, vol. 175, no. 13, pp. 2689-2708, 2018.

[83] M. Wang, D.-Q. Chen, L. Chen et al., "Novel RAS inhibitors poricoic acid ZG and poricoic acid $\mathrm{ZH}$ attenuate renal fibrosis via a $\mathrm{Wnt} / \beta$-catenin pathway and targeted phosphorylation of Smad3 signaling," Journal of Agricultural and Food Chemistry, vol. 66, no. 8, pp. 1828-1842, 2018.

[84] Y. Y. Yong, H. Chen and M. Wang. "Poricoic Acid A Is Used to Prepare Diuretic Drugs," CN106491622B [P/OL]. 201904-02].

[85] W. J. Ni, S. L. Yu, L. H. Zhang et al., "Virtual screening of hydrophilic effect of triterpenoids from Poria cocos," China Pharmaceuticals, vol. 28, no. 11, pp. 40-43, 2019.

[86] K. J. Ning, J. S. Yang, and P. P. Shi, "Observation of Poria cocos on diuretic effect in rabbits," Journal of Anhui Science and Technology Universit, vol. 26, no. 4, pp. 1-3, 2012.

[87] K. Wu, C. Guo, B. Wang, X. Wu, and W. Wang, "Antihepatotoxic benefits of Poria cocos polysaccharides on acetaminophen-lesioned livers in vivo and in vitro," Journal of Cellular Biochemistry, vol. 120, no. 5, pp. 7482-7488, 2019.

[88] C. Wang, X. Huo, L. Gao et al., "Hepatoprotective effect of carboxymethyl pachyman in fluorouracil-treated CT26bearing mice," Molecules, vol. 22, no. 5, p. 756, 2017.

[89] Y. C. Sun, W. L. Chang, S. F. Huang et al., "Pachymic acid stimulates glucose uptake through enhanced GLUT4 expression and translocation," European Journal of Pharmacology, vol. 648, no. 1-3, pp. 39-49, 2010.

[90] C. L. Huang, J. L. Zheng, F. L. Li et al., "Hypoglycemic effects of polysaccharides from Wolfiporia cocos (Schw.) Ryv. \& Cilbn in type II diabetic mice," Food Research and Development, vol. 37, no. 4, pp. 21-25, 2016.

[91] S.-S. Sun, K. Wang, K. Ma, L. Bao, and H.-W. Liu, “An insoluble polysaccharide from the sclerotium of Poria cocos improves hyperglycemia, hyperlipidemia and hepatic steatosis in ob/ob mice via modulation of gut microbiota," Chinese Journal of Natural Medicines, vol. 17, no. 1, pp. 3-14, 2019.

[92] P. Zhang, H. J. Hu, Y. Cheng et al., "Antioxidant activity of Poria cocos polysaccharides collected from different regions,"
Journal of Dongguan University of Technology, vol. 25, no. 1, pp. 63-67, 2018.

[93] L. S. Rak, L. Seulah, M. Eunjung et al., Chemistry-Bioorganic Chemistry; Findings from Sungkyunkwan University in Bioorganic Chemistry Reported (Bioactivity-Guided Isolation of Anti-inflammatory Triterpenoids from the Sclerotia of Poria cocos Using LPS-Stimulated Raw 264.7 Cells), Chemicals \& Chemistry, 2017.

[94] S. C. Qin, A. M. Wang, and R. Y. Li, "Effects of pachymic acid on inflammation, oxidative stress injury and apoptosis of SH-SY5Y cells induced by TNF- $\alpha$," Chinese Journal of Traditional Medical Science and Technology, vol. 26, no. 6, pp. 854-857, 2019.

[95] T. E. Liang, Y. Y. Liu, and X. Wang, "Study on mechanisms of Poria cocos polysaccharide regulating mast cell activation in rats with ulcerative colitis based on IL-33/ST2 signaling pathway," Chinese Journal of Immunology, vol. 36, no. 11, pp. 1324-1329+1337, 2020.

[96] H. Xu, Y. Wang, P. W. Jurutka et al., "16 $\alpha$-hydroxytrametenolic acid from Poria cocos improves intestinal barrier function through the glucocorticoid receptor-mediated PI3K/Akt/NF- $\kappa \mathrm{B}$ pathway," Journal of Agricultural and Food Chemistry, vol. 67, no. 39, pp. 10871-10879, 2019.

[97] H. H. Xiao, M. B. Zhang, Z. Q. Guo et al., "Study on mechanism poria in inhibiting contraction of small intestine in vitro," Modern Chinese Medicine, vol. 22, no. 9, pp. 1478-1484, 2020.

[98] K. Y. Song, Z. Y. Jiang, C. Q. Yan et al., "Experimental study on the effect of Radix codonopsis and Poria on intestinal flora in mice," Chinese Journal Clinical Pharmacology, vol. 27, no. 2, pp. 142-145, 2011.

[99] S.-H. Hu, G. Zhou, and Y.-W. Wang, "Tyrosinase inhibitory activity of total triterpenes and poricoic acid A isolated from Poria cocos," Chinese Herbal Medicines, vol. 9, no. 4, pp. 321-327, 2017.

[100] H. Lee and H. J. Cha, "Poria cocos Wolf extracts represses pigmentation in vitro and in vivo," Cellular and Molecular Biology, vol. 64, no. 5, pp. 80-84, 2018.

[101] C. F. Shi, C. Liu, and R. Y. Yang, "Study on application of Poria cocos for treatment of patients with dark skin pigmentation," Modern Chinese Medicine, vol. 19, no. 1, pp. 60-63, 2017.

[102] Y. Pang, S. Zhu, and H. Pei, "Pachymic acid protects against cerebral ischemia/reperfusion injury by the PI3K/Akt signaling pathway," Metabolic Brain Disease, vol. 35, no. 4, pp. 673-680, 2020.

[103] C. M. Yu, J. P. Li, and X. M. Hu, "Poria cocos peels inhibit epileptic activity," Chinese Traditional Patent Medicine, vol. 39, no. 6, pp. 1288-1290, 2017.

[104] Y. Cheng, Z. X. Ding, Y. Zhang et al., "Protective effects of different Poria extracts on mice with acute liver injury," Journal of Anhui University of Chinese Medicine, vol. 39, no. 4, pp. 73-77, 2020. 